\title{
On the accurate direct computation of the isothermal compressibility for normal quantum simple fluids: Application to quantum hard spheres.
}

\author{
Luis M. Sesé
}

Departamento de Ciencias y Técnicas Fisicoquímicas, Facultad de Ciencias, Universidad Nacional de Educación a Distancia, Paseo Senda del Rey 9, 28040 Madrid, Spain

A systematic study of the direct computation of the isothermal compressibility of normal quantum fluids is presented by analyzing the solving of the Ornstein-Zernike integral equation (OZ2) for the pair correlations between the path-integral necklace centroids. A number of issues related to the accuracy that can be achieved via this sort of procedure have been addressed, paying particular attention to the finite- $N$ effects and to the definition of significant error bars for the estimates of isothermal compressibilities. Extensive path-integral Monte Carlo computations for the quantum hard-sphere fluid (QHS) have been performed in the $(N, V, T)$ ensemble under temperature and density conditions for which dispersion effects dominate the quantum behavior. These computations have served to obtain the centroid correlations, which have been processed further via the numerical solving of the $O Z 2$ equation. To do so, Baxter-Dixon-Hutchinson's variational procedure complemented with Baumketner-Hiwatari's grand-canonical corrections have been used. The virial equation of state has also been obtained and several comparisons between different versions of the QHS equation of state have been made. The results show the reliability of the procedure based on isothermal compressibilities discussed herein, which can then be regarded as a useful and quick means of obtaining the equation of state for fluids under quantum conditions involving strong repulsive interactions.

Published in The Journal of Chemical Physics, vol. 136, pp. 244504/1-15 (2012)

email: $\underline{\text { msese@eccia.uned.es }}$ 


\section{INTRODUCTION}

Feynman's path integrals $(\mathrm{PI})^{1,2}$ have become a standard tool in the modern statistical mechanics studies of quantum condensed matter. Through the discretization of the PI particle closed paths in necklaces composed of beads in imaginary time, $\beta \hbar=\hbar / k_{B} T$, the use of path integral Monte Carlo (PIMC) and path integral techniques based on molecular dynamics has led to a deep understanding of static and dynamic quantum effects in the fluid and solid phases. ${ }^{3,21}$ In all of these applications, a very fruitful concept has turned out to be that of the PI necklace centroid, i.e. a centre of mass for the PI necklace. This concept has brought about developments in diverse directions, for example: a) quantum dynamics, ${ }^{11,16,19}$ b) quantum effective potentials, ${ }^{22}$ and c) fluid and solid structural features and related questions. ${ }^{23-31}$ There are some technical subtleties in the definition and use of centroids in the study of quantum dynamics, ${ }^{11,16}$ but these will not be dealt with in the present article which will only be concerned with equilibrium.

Of particular interest regarding the PI centroid correlations is the key role they play in computing, through calculations in Fourier space of the isothermal compressibility [i.e. $S(k=0)$ ], the equation of state of quantum fluids. ${ }^{29}$ This fact reveals some deep connections between classical and quantum statistical mechanics, and allows one to utilize the centroid concept at equilibrium in a very pragmatic way: it is a very useful device for counting number fluctuations. Prior work by this author over the past years has provided the required Ornstein-Zernike $(O Z n)$ framework, as well as a number of physically significant two- and three- body applications. The results so obtained were based on $(N, V, T)$ simulations and showed excellent agreement with experiments and other theoretical approaches. ${ }^{27-29,32-42}$ Clearly, there are other general methods to obtain isothermal compressibilities for quantum fluids, such as the evaluation of the fluctuations in the $(\mu, V, T)$-number of particles ${ }^{43,44}$ or in the $(N, P, T)$-volume. ${ }^{43,15(b)-(\mathrm{c}), 45}$ To carry out extensive grand ensemble simulations appears to be a daunting task in the quantum case. Besides, when performing quantum $(N, V, T)$ or $(N, P, T)$ simulations, there are two well-known finite-size effects affecting isothermal compressibilities which have not yet been analyzed in detail. One is 
the explicit effect that is associated with the fixing of $N=N_{S}$ particles in the simulation, and the other is the implicit effect that is related to the influence of the periodic boundary conditions. ${ }^{44}$ Far from the critical point and for a sufficiently large sample size $N_{S}$, the main influence on the simulation results is caused by the explicit effect, which becomes even more determining in the case of short-range interactions.

Given the equivalence of ensembles (far from the critical point) and the exactness of $O Z 2$ for the PI centroid pair correlations, the route already employed in preliminary works ${ }^{41,42}$ by this author may be a useful starting point to tackle the foregoing questions. This route is based on the OZ2 treatment of $(N, V, T)$ necklace centroid pair structures, which are adequately corrected with grand-canonical terms to deal with the finite- $N$ effects. Not only does this route provide very accurate fluid equations of state, as shown below, but also it is very fast in computational terms. It is then to be regarded as a useful and more efficient alternative to the usual virial pressure $p$ equation under extreme conditions (e.g. strong repulsive interactions present). Moreover, the "fine tuning" presented in this investigation is a necessary step which will be valuable for a wide range of further related purposes. One may mention, for instance, the general topic of the $O Z n$ equations $^{4,43,46}$ in which it is contained. This topic deals with quantum and classical static structure factors for fluids, ${ }^{27,41,42,46-48}$ the decay properties of the particle correlations, ${ }^{39}$ interfacial phenomena, etc. ${ }^{46(d)}$ Besides, the study of complex molecular fluids that may be represented by spherically symmetric potentials (e.g. fluid hydrogen isotopomers) ${ }^{29,47}$ can also benefit from this fine tuning.

The scope of this article will be that of homogeneous and isotropic quantum monatomic fluids at equilibrium in which diffraction effects dominate their behavior. Each particle $(i)$ will be then represented by a PI elastic necklace composed of $P$ beads with coordinates $\mathbf{r}_{i}^{t}, t=1,2, \ldots, P$. Adjacent beads in a necklace are separated in imaginary time by $\beta \hbar / P$ and linked by harmonic forces with spring constant $m P / \beta^{2} \hbar^{2}$ ( $m=$ particle mass). Note that the exact quantum limit is retrieved for $P \rightarrow \infty,{ }^{49}$ but at nonzero temperatures statistical convergence is assumed to occur for 
a finite $P$. The necklace centroid (CM) position vector of atom $i$ is thus conventionally defined as $\mathbf{R}_{C M, i}=P^{-1} \sum_{t} \mathbf{r}_{i}^{t}$, but note that as stated elsewhere ${ }^{19,29}$ the CM variables can be defined by the action on the actual system of an external field of constant strength. The set of $N \times P$ beads is ruled by $\exp \left[-\beta W_{N P}\right]$, where $W_{N P}$ is the "potential" of the sample which takes into account the foregoing bead-bead harmonic couplings, the interactions between "equal-time" beads in different necklaces, and, in the efficient versions, also corrections that yield faster convergences with the use of workable $P$ values (see below). ${ }^{3,10-12,49-53}$

The quantum hard-sphere fluid (QHS) has been selected as a probe to illustrate this study. The issues addressed in this work are the following: the influence of a thorough canonical structural sampling in real space over long run lengths; the overall assessment of the importance of grand-canonical corrections to deal with the finite- $N$ effects; and, also, the fixing of significant error bars for the isothermal compressibility results arising from $O Z 2$ applications. The outline of this article is as follows. Section II describes the basic theory, with an emphasis on the role played by external fields in the partition function of the quantum system. Section III contains the computational details and Section IV the results and their discussion. Finally, Section V collates the main conclusions of this work.

\section{THEORY}

\section{A. Statement of the problem}

The homogeneous and isotropic quantum fluid at equilibrium will be represented by a grand-canonical partition function $\Xi(\mu, V, T)$. When exchange can be neglected $\Xi$ reads as the following weighted summation of canonical partition functions ${ }^{4}$

$$
\Xi=\sum_{N \geq 0} \exp (\beta \mu N) \operatorname{Tr}\left\{\exp \left(-\beta H_{N}^{0}\right)\right\}=\sum_{N \geq 0} \frac{\exp (\beta \mu N)}{N !} \int d \mathbf{r}^{N}\left\langle\mathbf{r}^{N}\left|\exp \left(-\beta H_{N}^{0}\right)\right| \mathbf{r}^{N}\right\rangle
$$

where $\mu$ is the chemical potential, and $d \mathbf{r}^{N}=d \mathbf{r}_{1} d \mathbf{r}_{2} \ldots d \mathbf{r}_{N}$ and $H_{N}^{0}=T+U$ are the multidimensional volume element and the Hamiltonian for $N$ structureless particles, respectively. 
The average number density will be denoted by $\rho_{N}=\langle N\rangle / V$. Note that no restriction on the form of the interactions contained in the $U$ term has been imposed, and $U$ may take any significant many-body expression. It has to be stressed that the indistinguishability factors $N$ ! come from the symmetry properties of the complete canonical partition functions, from which one obtains Eq. (1) by retaining only the identity permutations when exchange effects can be neglected. ${ }^{2,4}$ Boltzmann statistics is then utilized to describe the system and to deal with quantum dispersion effects. In the study of fluids the $N$ ! factors are crucial in that they guarantee the correct transition quantum $\rightarrow$ classical in statistical mechanics ( $\hbar$ factors also appear in the latter case). ${ }^{2,43}$ In this regard, also note that for the equilibrium calculations of non-thermal properties in the canonical or the isothermal-isobaric ensembles, the corresponding factor $N$ ! may be omitted, as it cancels out when computing averages.

By considering the effect of an external field acting as $\Psi=\sum_{i} \Psi\left(\mathbf{r}_{i}\right)$, after applying $P$ times the product property of the canonical density operator one finds

$$
\Xi=\sum_{N \geq 0} \frac{\exp (\beta \mu N)}{N !} \int \prod_{t=1}^{P}{ }^{\prime} d \mathbf{r}^{N, t}\left\langle\mathbf{r}^{N, t}\left|\exp \left(-\beta H_{N}^{0} / P-\beta \Psi_{N} / P\right)\right| \mathbf{r}^{N, t+1}\right\rangle
$$

where $\mathbf{r}^{N, 1} \equiv \mathbf{r}^{N}$ above, $d \mathbf{r}^{N, t}=d \mathbf{r}_{1}^{t} d \mathbf{r}_{2}^{t} \ldots d \mathbf{r}_{N}^{t}$, and the primed product implies $P+1 \equiv 1$ when this occurs in the matrix element. $\Psi$ must vanish at the boundaries of the system and be compatible with the existence of eigenstates. Application of the approximation ${ }^{53(c)}$

$$
\exp \left(-\beta H_{N}^{0} / P-\beta \Psi_{N} / P\right) \approx \exp \left(-\beta \Psi_{N} / 2 P\right) \exp \left(-\beta H_{N}^{0} / P\right) \exp \left(-\beta \Psi_{N} / 2 P\right)
$$

which is accurate up to $O\left(P^{-3}\right)$ yields the discretized PI partition function

$$
\Xi \approx \Xi_{P}=\sum_{N \geq 0} \frac{\exp (\beta \mu N)}{N !} \int \prod_{t=1}^{P} d^{\prime} \mathbf{r}^{N, t}\left\langle\mathbf{r}^{N, t}\left|\exp \left(-\beta H_{N}^{0} / P\right)\right| \mathbf{r}^{N, t+1}\right\rangle \times \exp \left(-\frac{\beta}{P} \sum_{i=1}^{N} \sum_{t=1}^{P} \Psi\left(\mathbf{r}_{i}^{t}\right)\right)
$$

By defining the external field as one of constant strength $\mathbf{f}, \Psi_{F}=\sum_{i} \mathbf{f} . \mathbf{r}_{i}$, Eq. (4) can be rewritten for the actual system utilizing the coordinates of the PI centroids in the field factor 


$$
\Xi_{P}=\sum_{N \geq 0} \frac{\exp (\beta \mu N)}{N !} \int \prod_{t=1}^{P} d \mathbf{r}^{N, t}\left\langle\mathbf{r}^{N, t}\left|\exp \left(-\beta H_{N}^{0} / P\right)\right| \mathbf{r}^{N, t+1}\right\rangle \times \prod_{i=1}^{N} d \mathbf{R}_{i} \delta\left(\mathbf{R}_{i}-\mathbf{R}_{C M, i}\right) \times \exp \left(-\beta \sum_{i=1}^{N} \Psi_{F}\left(\mathbf{R}_{i}\right)\right)
$$

The physical significance of the process sketched above is guaranteed by the non-negativeness of the density matrix at all temperatures in the coordinate representation and also by Trotter's formula, ${ }^{49}$ which makes the raw primitive approximation for well-behaved operators ${ }^{6}$

$$
\exp (-\beta(T+U+\Psi) / P) \approx \exp (-\beta T / P) \exp (-\beta U / P) \exp (-\beta \Psi / P)
$$

exact in the limit $P \rightarrow \infty$. It is worthwhile insisting on the fact that the matrix elements contained in Eq. (5) are nonnegative quantities, which will be defined in terms of all the positions $\mathbf{r}^{N, t}$ by applying path-integral techniques. The optimum number $P$ guaranteeing statistical convergence for properties is assumed hereafter.

Note that the appearance of the centroids $\mathbf{R}_{C M, i}=P^{-1} \sum_{t} \mathbf{r}_{i}^{t}$ in Eq. (5) is only related to the consideration of the external field of constant strength in the actual quantum partition function. In this connection, the PI centroids are functions of the bead coordinates and, as such, one can evaluate averages related to these variables [e.g. the two-body correlation $\rho_{N}^{2} g_{C M}\left(R_{12}\right)$ ] when working with the system at $\Psi_{F}=0$. On the other hand, the foregoing development gives a precise physical meaning to the PI centroids at equilibrium. ${ }^{29}$ It is here where the use of the grand ensemble becomes decisive. Eq. (5) is a classical-like partition function which, through functional differentiation with respect to $\Psi_{F}(\mathbf{R})$, yields the whole range of correlation functions between the PI centroids of the fluid and their related response functions in Fourier space. The first two functional derivatives are

$$
\begin{aligned}
& -k_{B} T \frac{\delta \ln \Xi_{P}\left(\Psi_{F}\right)}{\delta \Psi_{F}\left(\mathbf{R}_{1}\right)}=\rho_{N, C M}^{(1)}\left(\mathbf{R}_{1} ; \Psi_{F}\right) \\
& -k_{B} T \frac{\delta \rho_{N, C M}^{(1)}\left(\mathbf{R}_{1} ; \Psi_{F}\right)}{\delta \Psi_{F}\left(\mathbf{R}_{2}\right)}=\rho_{N, C M}^{(2)}\left(\mathbf{R}_{1}, \mathbf{R}_{2} ; \Psi_{F}\right)-\rho_{N, C M}^{(1)}\left(\mathbf{R}_{1} ; \Psi_{F}\right) \rho_{N, C M}^{(1)}\left(\mathbf{R}_{2} ; \Psi_{F}\right)+\rho_{N, C M}^{(1)}\left(\mathbf{R}_{1} ; \Psi_{F}\right) \delta\left(\mathbf{R}_{1}-\mathbf{R}_{2}\right)
\end{aligned}
$$


where $\rho_{N, C M}^{(1)}$ and $\rho_{N, C M}^{(2)}$ are the one-body and the two-body spatial correlation functions, which in a weak field can be approximated by the zero field functions $\rho_{N}=\langle N\rangle / V$ and $\rho_{N}^{2} g_{C M}\left(R_{12}\right)$, respectively. The linear response in the field $\Psi_{F}$ for the PI centroids is the static structure factor ${ }^{29}$ (Appendix I)

$$
S_{C M}(k)=1+\rho_{N} \int d \mathbf{R}_{12}\left(g_{C M}\left(R_{12}\right)-1\right) \exp \left(-i \mathbf{k} \cdot \mathbf{R}_{12}\right)
$$

The latter cannot be measured directly in an experiment because the field couples with actual particles. However, $S_{C M}$ can be connected to measurable properties obtainable with scattering techniques. $^{32-34,47}$

The exact $O Z 2$ equation for PI centroid correlations turns out to be formally the same ${ }^{29}$ as that of a classical fluid which is composed of structureless particles (Appendix I). ${ }^{43,46}$ In the absence of the external field (homogeneity and isotropy are assumed) it reads as

$$
h_{C M}\left(R_{12}\right)=c_{C M}\left(R_{12}\right)+\rho_{N} \int d \mathbf{R}_{3} h_{C M}\left(R_{13}\right) c_{C M}\left(R_{23}\right)
$$

where $h_{C M}=g_{C M}-1$ and $c_{C M}$ is the direct pair correlation function. Note that the complete set of classical-like equations $\operatorname{OZn}(n=2,3, \ldots)$ for the PI centroids can also be exactly defined..$^{29,41}$ This fact extends the usefulness of these PI variables to deal with correlations between particles beyond the pair level, either at zero field or under a constant force field. With the use of Eq. (9) one writes in Fourier space ${ }^{29}$

$$
S_{C M}(k)=\left(1-\rho_{N} c_{C M}(k)\right)^{-1}
$$

where $c_{C M}(k)$ is the Fourier transform of $c_{C M}\left(R_{12}\right)$. Besides, fluctuation theory yields the central equation of state $^{29}$

$$
S_{C M}(k=0)=\frac{\left\langle N^{2}\right\rangle-\langle N\rangle^{2}}{\langle N\rangle}=\rho_{N} k_{B} T \chi_{T}=k_{B} T\left(\frac{\partial \rho_{N}}{\partial p}\right)_{T}
$$

where $\chi_{T}$ is the isothermal compressibility. Given that the linear response radial function between pairs of PI centroids (i.e. the pair radial function $g_{C M}$ at $\Psi_{F}=0$ ) is exactly linked to the 
isothermal compressibility, that function can be determined by utilizing the propagator in the absence of the external field, $\left\langle\mathbf{r}^{N, t}\left|\exp \left(-\beta H_{N}^{0} / P\right)\right| \mathbf{r}^{N, t+1}\right\rangle$. Therefore, the particular form to be used for this propagator is now a matter of convenience (e.g. primitive, pair actions, etc.), as the problem has been transferred to the isolated fluid (linear response). In this regard, the rates of $P$ convergence and other features ${ }^{11}$ should be taken into consideration when planning the computations to be carried out. Besides, one can always fix the number $P$ of beads which is best suited for the purpose of all these calculations, and no inconsistencies between the above centroid derivation and the final isolated system calculations arise.

It is worth remarking that Eq. (11) shows no explicit dependence on the interactions contained in $U$, and hence it is completely general. ${ }^{56}$ This fact stems directly from the particle number fluctuations and makes Eq. (11) a powerful tool. As a consequence, the final centroid scheme, Eqs. (9)-(11), can also be applied with no changes when the Hamiltonian includes interactions beyond the atom-atom level. Note that, the action of the field as obtained through Eqs. (3)-(5), and also the $\Psi$-functional manipulations [Eqs. (7) and Appendix I], are clearly independent of the interactions between the particles. ${ }^{28,29}$ These interactions will only affect the isolated system $H^{0}$ calculations. In connection with this, recall that: a) $U$ is diagonal in the coordinate representation; and b) if higher-order interactions were taken into account, their effects would contribute to shape the $g_{C M}$ pair radial function which defines unambiguously $\chi_{T}$.

Once the $g_{C M}\left(R_{12}\right)$ function has been computed, the solving of Eq. (9) can be achieved in a very convenient way with Baxter's partition ${ }^{54}$ and Dixon-Hutchinson's variational procedure ${ }^{55}$ a method that will be termed $\mathrm{BDH}$ for brevity. In sharp contrast to other OZ2 methods, ${ }^{43}$ the numerical application of $\mathrm{BDH}$ does not depend explicitly on the potential $U$ acting between the particles involved, which makes BDH most suitable for dealing with a wide range of situations, in particular PI-CM correlations. It will be assumed in what follows that the implicit finite-size effects are negligible and that it will suffice to correct $g_{C M}\left(R_{12}\right)$ for the explicit finite-size effects (hereafter finite- $N$ effects). This task can also be accomplished with the use of BDH and although 
full details of the BDH implementation in the canonical ensemble can be found elsewhere, ${ }^{32,35,36}$ a short account of this method is needed for the current purposes.

\section{B. BDH method}

By assuming the existence of a cut-off at a distance $R$, such that $c\left(R_{12}\right)=0$ for $R_{12} \geq R$, and that $h\left(R_{12}\right)$ is known for $0 \leq R_{12} \leq R$, Eq. (9) can be transformed into the pair of coupled equations $^{54}$

$$
\begin{aligned}
R_{12} h\left(R_{12}\right) & =-Q^{\prime}\left(R_{12}\right)+2 \pi \rho_{N} \int_{0}^{R} d r\left(R_{12}-r\right) h\left(\left|R_{12}-r\right|\right) Q(r) ; R_{12}>0 \\
R_{12} c\left(R_{12}\right) & =-Q^{\prime}\left(R_{12}\right)+2 \pi \rho_{N} \int_{R_{12}}^{R} d r Q\left(r-R_{12}\right) Q^{\prime}(r) ; \quad 0<R_{12}<R \\
& =0 ; \quad R_{12}>R
\end{aligned}
$$

$Q$ is an auxiliary function continuous at $R_{12}=R$, and such that $Q\left(R_{12}\right)=0$ for $R_{12} \geq R$. It is related to $Q^{\prime}=d Q / d r$ through

$$
Q\left(R_{12}\right)=-\int_{R_{12}}^{R} Q^{\prime}(r) d r
$$

Eq. (12a) is an integral equation which gives $Q^{\prime}$, and via Eq. (12b) $Q, c\left(R_{12}\right)$ and $c(k)$ can be obtained, with the proviso that an extrapolation to obtain $c\left(R_{12}\right)=0$ has been carried out [also assumed is $c(R)=0]$.

The foregoing equations contain the basic results arising from the Wiener-Hopf factorization put forward by Baxter for dealing with disordered fluids through Eq. (9). Although, for classical hard spheres the above equations yield the Percus-Yevick exact solution, Baxter's insightful development needs some extra conditions and further elaboration to obtain accurate results in the general case.

In this regard, Baxter already noted that specifically

$$
Q(k=0)=\left\{1-2 \pi \rho_{N} \int_{0}^{R} d r \exp (i k r) Q(r)\right\}_{k=0}>0
$$

is a necessary condition related to some intricacies of the convergence of integrals in the auxiliary complex plane $k=x+i y$, and that can be related to the isothermal compressibility as 
$S(k=0)=\{Q(k=0)\}^{-2}=\left\{1-\rho_{N} \int_{0}^{R} d \mathbf{r} c(r)\right\}^{-1}$

Therefore, Eq. (14a) is a compulsory test for the solutions arising from Eqs. (12). Furthermore, Dixon and Hutchinson ${ }^{55}$ pointed out two additional problems with Eqs. (12): i) that arbitrary $R$ values do not necessarily preserve the continuity of $h\left(R_{12}\right)$ at $R_{12}=R$ (continuity of the potential is assumed); and ii) that the behavior of Eqs. (12) at small $R_{12}$ values may produce highly inaccurate results for $c\left(R_{12}\right)$. The solutions that the latter authors gave to i) and ii) are summarized in what follows:

a) The condition for the cut-off distance $R$ to preserve the $h$ continuity is

$Q^{\prime}(R)=0$, or, at worst, $\left[Q^{\prime}(R) / R\right]^{2}=$ minimum

These proper cut-off $R$ values will be denoted by $R_{Z}$ (zeros) hereafter. In this connection, one notes that although the very existence of these zeros has not been proved analytically, previous work by the present author indicates that they actually show up in these calculations. There may be one or, in general, more than one $R_{Z},{ }^{32,35,36}$ and the solution to this cut-off $R$ problem is not expected to be unique.

b) The procedure giving $Q^{\prime}\left(R_{12}\right)$ is based on the least-squares minimization of a non-negative merit function $F$ which takes into account the behavior of Eqs. (12) for $r \geq 0$. By using a discretization of the range $0 \leq r \leq R$ to $n$ points $\left\{r_{i}\right\}_{i=1, n}$, one obtains a linear system in the associated unknowns $\left\{Q_{i}^{\prime}\right\}_{i=1, n}$

$\left(\frac{\partial F}{\partial Q_{i}^{\prime}}\right)_{R}=\sum_{j=1}^{n} a_{i j} Q_{j}^{\prime}+b_{i}=0 ; \quad i=1,2,3, \ldots, n$

where $a_{i j}$ and $b_{i}$ depend on the $\left\{Q_{i}\right\}_{i=1, n}$ values. The solution of this system may thus be found iteratively. Reliable limits for this $F$ minimization, typically $5 \times 10^{-6} \leq F \leq 10^{-4}$, are known to be reached generally in 300 iterations at the most. ${ }^{32-36}$ From a practical point of view, the determination of the $R_{Z}$ zeros can be accomplished by analyzing a series of trial cut-off distances 
$\left\{R_{T}\right\}$, within $\sigma<R<L / 2$ with $L$ being the length of the simulation cubic box. The parameter $\sigma$ is a short-range distance that may be set arbitrarily, although a good choice is a hard-sphere diameter for the particles. By using the tabulation $\left\{R_{T}, Q^{\prime}\left(r ; R_{T}\right)\right\}$ and applying Eqs. (12)-(14) one obtains the set of different possible solutions $\left\{R_{Z}, Q^{\prime}\left(r ; R_{Z}\right), c\left(r ; R_{Z}\right)\right\}_{Z}$. The latter set poses new problems, as one is forced to analyze these results further to obtain a well-defined answer to this problem.

\section{Further problems}

If the range of distances, determined in the $(N, V, T)$ ensemble, for $h\left(R_{12}\right)$ is $0 \leq R_{12} \leq L / 2$, one might expect to have a fine description by using the results corresponding to the $R_{Z}$ zero closest to $L / 2$. However, although the longer the $R_{Z}$ the better the results for $c\left(R_{12}\right)$ would seem, the rapid decay of the latter function makes other lower $R_{Z}$ as significant as, or even more than, the longest one. Note that PIMC canonical simulations bear the burden of the $O(1 / N)$ asymptotic behavior in the pair radial correlation functions, ${ }^{44,56}$ which will have a non-negligible impact connected to the finite- $N$ effects on the results. To avoid the daunting task of carrying out extensive grand ensemble simulations, the $(N, V, T)$ calculations, ${ }^{57}$ appropriately treated, can provide a broad solution not only to the computation of $\chi_{T}$, but also to the fixing of static structure factors $S(k)$ out of any direct PI simulation scheme.

Working in the canonical ensemble and with a sufficiently long $L$, the $R_{Z}$ zeros relatively close to $L / 2$ are likely to be affected by the finite- $N$ effects, which can deteriorate the accurate computation of the low- $k$ range of wave vectors, and thus of the $\chi_{T}$ estimates. Note that this situation would be much worse if the direct simulation of $S(k=0)$ was undertaken, owing to the drastic limitation imposed by the finite $L$ on small wave vectors. ${ }^{6,43}$ On the other hand, lower $R_{Z}$ zeros contained within a mid-upper range of distances are in general expected to be much less sensitive to finite- $N$ effects. In fact, in most of the calculations carried out so far with the BDH 
method $^{30(\mathrm{a}), 38,41}$ it has been observed that the $\chi_{T}$ computed values are distributed about a mean value along a mid-upper range of zeros ( $K$ in number). On the other hand, the complementary measure of the inclusion of grand-canonical corrections to the canonical pair correlation function $^{44,58}$ improves its long distance behavior, while leaving unaltered the BDH basic features. ${ }^{41,42}$ All of this suggests that, after the application of the combined effect BDH +grandcanonical corrections to the pair correlation function, one should employ some sort of average over an appropriately defined mid-upper subset of the $\left\{R_{Z}, \chi_{T}\right\}$ values obtained to produce a final estimate of $\chi_{T}$. Last but not least, there still remains the difficult issue of fixing reliable error bars for the isothermal compressibility computed in this way that involves integral equations.

Previous works by this author have dealt to some extent with all the foregoing problems, as yet the error bar issue remains unsolved. ${ }^{30(a), 38}$ A proper and consistent treatment of the error bars is central to assess the relative numerical accuracy of the current $\chi_{T}$ route as compared to that of the standard $(N, V, T)$ virial pressure method. The following are the proposed actions to reach a more precise answer to this complex situation at a given state point.

\section{Proposed actions}

a) The first most obvious action is two-fold and is related to the initial PIMC calculations: the use of a large sample size $N_{S}$ (number of actual particles), and the increase of the run length. By so doing, one could reduce the finite- $N$ effects rendering them negligible from a practical point of view, and also obtain improved results for the fine long distance details which define the low- $k$ behavior.

b) The second action is intended to alleviate the burden of using a large $N_{S}$ and consists in including grand-canonical corrections to the canonical centroid pair radial correlation function. It is expected then that a compromise between the use of a moderately large sample size and the "harnessing" of the significant part of the finite- $N$ effects may be reached. A straightforward and valuable way to achieve this end is the iterative process put forward by Baumketner and Hiwatari, ${ }^{58}$ which treats the canonical output $g_{C M}\left(R_{12}\right)$ as 
$g_{C M}^{(m)}\left(R_{12}\right)=g_{C M}\left(R_{12}\right)\left\{1+\frac{S_{C M}^{(m-1)}(k=0)}{N_{S}}\right\} ; m=1,2,3, \ldots, m_{\max }$

When convergence is reached the process Eq. (17a) yields the approximation to the grand canonical function of the corresponding open system

$g_{C M}^{G C}\left(R_{12}\right) \cong g_{C M}^{\left(m_{\max }\right)}\left(R_{12}\right)$

This method (GC) assumes that there is an independent way to obtain $S_{C M}(k=0)$. The zero step is given by $g_{C M}\left(R_{12}\right)$ and its BDH associated value $S_{C M}^{(0)}(0)$; the first step uses $g_{C M}\left(R_{12}\right)$ and $S_{C M}^{(0)}(0)$ to obtain the corrected function $g_{C M}^{(1)}\left(R_{12}\right)$, with which $S_{C M}^{(1)}(0)$ can be fixed; and so on. For normal quantum fluid state points, and with the use of $\mathrm{BDH}$ to obtain $S_{C M}(k=0)$, the GC process is known to converge for practical purposes in a few iterations $(m \leq 5)$. A stable final pair function is produced and is taken as the approximation to the grand canonical function $g_{C M}^{G C}\left(R_{12}\right)$. This process is also known to yield physically significant results not only for the isothermal compressibility, but also for other properties when applied to the appropriate pair function (e.g. instantaneous correlations and static structure factor). ${ }^{41,42}$

c) The third action is related to the definition of the error bars in $\chi_{T}$ which arise from the statistical uncertainties in the PIMC mean canonical function $g_{C M}\left(R_{12}\right)$, or in the mean $h_{C M}\left(R_{12}\right)$. The latter will de denoted by $\left\langle h_{C M}\left(R_{12}\right)\right\rangle$ hereafter. Given the data $\left\{R_{Z}, \chi_{T}\right\}$, a statistical population of significant values for $R_{Z}$ and another statistical population of $\chi_{T}$ are assumed to occur. If the total run length is split into $M_{B}$ equal-length simulation blocks, each of which giving the subaverage $\alpha$-block structure $\bar{h}_{C M, \alpha}\left(R_{12}\right)$, one finds the following structural estimates ${ }^{59}$

$$
\begin{aligned}
& \left\langle h_{C M}\left(R_{12}\right)\right\rangle=\frac{1}{M_{B}} \sum_{\alpha=1}^{M_{B}} \bar{h}_{C M, \alpha}\left(R_{12}\right) \\
& s^{2}\left(\bar{h}_{C M}\left(R_{12}\right)\right)=\frac{1}{M_{B}-1} \sum_{\alpha=1}^{M_{B}}\left\{\bar{h}_{C M, \alpha}\left(R_{12}\right)-\left\langle h_{C M}\left(R_{12}\right)\right\rangle\right\}^{2}
\end{aligned}
$$


The analysis of the propagation of the variances given in Eq. (18b) throughout the complete iterative procedure appears to be a formidable task. There are many stages and, what is more, there is also the fact that an integral equation relates the unknown solution to its values over the region of definition, including the boundary (which is defined by $R_{Z}$ ). Therefore, the search for a straightforward, yet physically significant, alternative to obtain error bars in the final results for $\chi_{T}$ seems worth pursuing.

In this connection, a possible alternative consists in performing the following three main steps (see Appendix II for the detailed procedure):

i) Analyze with $\mathrm{BDH}+\mathrm{GC}$ the mean structure $\left\langle h_{C M}\left(R_{12}\right)\right\rangle$ and compute the global estimates

$$
\begin{aligned}
& \left\langle\chi_{T}\right\rangle=\frac{1}{K^{G C}} \sum_{v=1}^{K^{G C}} \chi_{T, v}=\frac{\left\langle S_{C M}(0)\right\rangle}{\rho_{N} k_{B} T} \\
& s^{2}\left(\left\langle\chi_{T}\right\rangle\right) \approx s^{2}\left(\chi_{T, v}\right)=\frac{1}{\left(K^{G C}-1\right)} \sum_{v=1}^{K^{G C}}\left(\chi_{T, v}-\left\langle\chi_{T}\right\rangle\right)^{2}
\end{aligned}
$$

where $K^{G C}$ stands for the number of significant mid-upper zeros obtained, which define the final mid-upper range of zeros $R_{Z 1} \leq R_{Z, v} \leq R_{Z K^{G C}}$. The latter is identified by the slight oscillations of $\chi_{T, v}$. At each GC $m$ step use the average over the $S_{C M, v}^{(m-1)}(0)$ in Eqs. (17).

ii) Analyze with $\mathrm{BDH}+\mathrm{GC}$ every block structure $\bar{h}_{C M, \alpha}\left(R_{12}\right)$ and compute the block estimates $\bar{\chi}_{T, \alpha}$ and $s^{2}\left(\chi_{T, \eta(\alpha)}\right)$ in an analogous fashion to Eqs. (19) and (20). Next, compute the pooled quantities that take into account the different numbers $K^{G C}(\alpha)$ of zeros per block obtained

$$
\begin{gathered}
\bar{\chi}_{T}=\frac{\sum_{\alpha=1}^{M_{B}} K^{G C}(\alpha) \bar{\chi}_{T, \alpha}}{\sum_{\alpha=1}^{M_{B}} K^{G C}(\alpha)}=\frac{\bar{S}_{C M}(0)}{\rho_{N} k_{B} T} \\
s^{2}\left(\bar{\chi}_{T}\right)=\frac{\sum_{\alpha=1}^{M_{B}}\left(K^{G C}(\alpha)-1\right) s^{2}\left(\chi_{T, \eta(\alpha)}\right)}{\left(\sum_{\alpha=1}^{M_{B}} K^{G C}(\alpha)\right)\left(\sum_{\alpha=1}^{M_{B}} K^{G C}(\alpha)-M_{B}\right)}
\end{gathered}
$$


iii) Compute the final estimates of the error bars for $\left\langle\chi_{T}\right\rangle$ as

$$
\varepsilon\left(\left\langle\chi_{T}\right\rangle\right)=\left|\left\langle\chi_{T}\right\rangle-\bar{\chi}_{T}\right|+\sqrt{s^{2}\left(\left\langle\chi_{T}\right\rangle\right)+s^{2}\left(\bar{\chi}_{T}\right)}
$$

where independence between the results obtained in i) and in ii) is applied.

Eq. (23) is an estimator that tends to the essential dispersion $s\left(\left\langle\chi_{T}\right\rangle\right)$ with the gathering of more and more statistics, since the other contributions will tend to zero (see Appendix III). The rationale behind $s^{2}\left(\left\langle\chi_{T}\right\rangle\right)$ is that it is expected to diminish by enhancing statistics. This behavior would result from the better definition of the significant mid-upper range of $R_{Z, v}$ zeros and of their associated $\chi_{T, v}$. When employing sufficiently long runs whether each of the $\chi_{T, v}$ tends to the same common value, i.e. $\left\langle\chi_{T}\right\rangle$, or it is their average which $\left\langle\chi_{T, v}\right\rangle \rightarrow\left\langle\chi_{T}\right\rangle$, is a matter difficult to foresee. It might even occur that the whole $\left\{\chi_{T, v}\right\}$ set showed steady, though very small, oscillations about the value $\left\langle\chi_{T}\right\rangle$, which is the effect to be observed in normal practice. In any case, the actual behavior can be extracted from the computations, and $s\left(\left\langle\chi_{T}\right\rangle\right)$ and $\varepsilon\left(\left\langle\chi_{T}\right\rangle\right)$ will be useful as long as they remain sufficiently small in comparison to $\left\langle\chi_{T}\right\rangle$. An indication of the reliability of the whole process can be obtained if the expected relationship $s^{2}\left(\left\langle\chi_{T}\right\rangle\right)<s^{2}\left(\bar{\chi}_{T}\right) \sum_{\alpha} K^{G C}(\alpha)$ holds, which would mean that the precision in the fixing of $\chi_{T}$ becomes higher by enhancing statistics. A more stringent test is given by the comparisons $s^{2}\left(\left\langle\chi_{T}\right\rangle\right)<s^{2}\left(\chi_{T, \eta(\alpha)}\right), \alpha=1,2, \ldots, M_{B}$. In this regard, note that one is only allowed to employ the $t$-Student distribution [step i)] and Fisher $F$ tests [step ii)] if the involved populations $\left\{\chi_{T}\right\}$ follow Gaussian behavior. However, such behavior does not seem to be the general case (see below), and one should resort to non-parametric tests (e.g. sign test) to carry out such comparisons. ${ }^{59}$ 


\section{COMPUTATIONAL DETAILS}

The calculations carried out in this paper have been based on PIMC- $(N, V, T)$ simulations, for which ( $P$ convergence is reached) the canonical partition function is given by

$$
Z_{N}=\operatorname{Tr}\left(\exp \left(-\beta H_{N}^{0}\right)\right) \approx Z_{N, P}=(N !)^{-1}\left(\frac{m P}{2 \pi \beta \hbar^{2}}\right)^{3 N P / 2} \int \prod_{i=1}^{N} \prod_{t=1}^{P} d \mathbf{r}_{i}^{t} \exp \left[-\beta W_{N, P}\right]
$$

The necklace centroid pair structures $g_{C M}$ are defined through $(\langle\ldots\rangle=$ canonical average $)$

$$
\rho_{N}^{2} g_{C M}\left(R_{12}\right)=\left\langle\sum_{i \neq j} \delta\left(\mathbf{R}_{C M, i}-\mathbf{q}_{1}\right) \delta\left(\mathbf{R}_{C M, j}-\mathbf{q}_{2}\right)\right\rangle ; \quad R_{12}=\left|\mathbf{q}_{1}-\mathbf{q}_{2}\right|
$$

For completeness, two more significant pair radial correlations have also been computed. These are: $g_{E T}(r)$ which stands for the instantaneous pair correlation function $(E T=$ "equal imaginary time")

$$
\rho_{N}^{2} g_{E T}\left(r_{12}\right)=\left\langle\frac{1}{P} \sum_{i \neq j} \sum_{t=1}^{P} \delta\left(\mathbf{r}_{i}^{t}-\mathbf{q}_{1}\right) \delta\left(\mathbf{r}_{j}^{t}-\mathbf{q}_{2}\right)\right\rangle ; r_{12}=\left|\mathbf{q}_{1}-\mathbf{q}_{2}\right|
$$

and the total continuous linear response structure $G_{T L R}(r)$ which is the overall bead-bead average

$$
\begin{aligned}
& \left(P \rho_{N}\right)^{2} G_{T L R}\left(r_{12}\right)=\left(P \rho_{N}\right)^{2}\left(s_{S C}\left(r_{12}\right)+g_{P L R}\left(r_{12}\right)\right)= \\
& \left\langle\sum_{i=1}^{N} \sum_{t \neq t^{\prime}} \delta\left(\mathbf{r}_{i}^{t}-\mathbf{q}_{1}\right) \delta\left(\mathbf{r}_{i}^{t^{\prime}}-\mathbf{q}_{2}\right)+\sum_{i \neq j} \sum_{t(i)=1}^{P} \sum_{t^{\prime}(j)=1}^{P} \delta\left(\mathbf{r}_{i}^{t}-\mathbf{q}_{1}\right) \delta\left(\mathbf{r}_{j}^{t^{\prime}}-\mathbf{q}_{2}\right)\right\rangle .
\end{aligned}
$$

Both types of radial functions can be determined through experiments and shape the actual linear response functions of the system to the action of an external field. For $g_{E T}(r)$ the field is of a localizing type (i.e. defined by a singular $\delta$ potential, as in elastic neutron scattering), whereas for $G_{T L R}(r)$ the field is a continuous function of the position. ${ }^{27-29}$ The static response functions read as

$$
\begin{aligned}
& S_{E T}(k)=1+\rho_{N} \int d \mathbf{r}_{12} h_{E T}\left(r_{12}\right) \exp \left(-i \mathbf{k} \cdot r_{12}\right) \\
& S_{T L R}(k)=P^{-1}+\rho_{N} \int d \mathbf{r}_{12}\left(s_{S C}\left(r_{12}\right)+h_{P L R}\left(r_{12}\right)\right) \exp \left(-i \mathbf{k} \cdot r_{12}\right)
\end{aligned}
$$

These two functions, together with $S_{C M}(k)$, can be connected via "sum rules" to the dynamic structure factor $S(k, \omega){ }^{47,48,60}$ 


\section{A. PIMC simulations}

The QHS system (classical collision parameter $\sigma$ ) has been studied at 45 state points for ranges of fluid density conditions, $0.1 \leq \rho_{N}^{*}=\rho_{N} \sigma^{3}$, compatible with the selected de Broglie wavelengths $\lambda_{B}^{*}=h /\left(2 \pi m k_{B} T \sigma^{2}\right)^{1 / 2}: \lambda_{B}^{*}=0.2,0.4,0.6,0.8$, and $2 .{ }^{61}$ The increments in density have been taken as $\Delta \rho_{N}^{*}=0.1,0.025$, and 0.015 , depending upon the region considered. Within the range $\lambda_{B}^{*} \leq 0.8$ state points inside the metastability regime ${ }^{30(a)}$ have also been analyzed with a twofold purpose. First, to investigate the application of $\mathrm{BDH}+\mathrm{GC}$ under metastable conditions, as some numerical problems were observed in previous works. Secondly, to obtain better results for the fluid which allow one to study further the fluid-solid phase transitions. The onset of the fluid metastablity has been taken as follows: ${ }^{30(a)}$ for $\lambda_{B}^{*}=0.2, \rho_{N}^{*} \approx 0.75$; for $\lambda_{B}^{*}=0.4, \rho_{N}^{*} \approx 0.665$; for $\lambda_{B}^{*}=0.6, \rho_{N}^{*} \approx 0.55 ;$ for $\lambda_{B}^{*}=0.8, \rho_{N}^{*} \approx 0.515$. The computations along $\lambda_{B}^{*}=2$ at $\rho_{N}^{*}=0.1,0.2$, and 0.3 have been carried out for completeness. Although the degeneracy parameter along $\lambda_{B}^{*}=2$, $\gamma=\lambda_{B}^{3} \rho_{N}$, is close to or greater than unity, this isotherm can be mapped onto fluid helium-4 at $T \approx 4 K$, as discussed by Runge and Chester. ${ }^{30(\mathrm{~b})}$ Accordingly, exchange interactions have also been neglected, the purpose of these computations being to investigate the application of $\mathrm{BDH}+\mathrm{GC}$ under very strong diffraction effects in the stable fluid phase.

For the path-integral $N \times P$ hard-sphere system the pair action put forward by Cao and Berne $^{51}$ has been selected. Apart from the PI centroid correlations the main targets have been the instantaneous function $g_{E T}(r)$ and the virial $p$ pressure, which is given by

$$
p=\frac{2 E}{3 V}+\frac{\rho_{N}^{2} \pi \sigma^{3} \hbar^{2}}{3 m}\left[\frac{d^{2} g_{E T}(r)}{d r^{2}}\right]_{r=\sigma+}
$$

where $E$ stands for the internal energy and the second term is known as Fierz's term. ${ }^{62}$ Grand canonical corrections Eqs. (17) to the foregoing calculation are expected to be negligible. This fact can be understood by realizing that the energy contribution decays exponentially with the distance, 
and that Fierz's term is an effect localized at "classical contact". The reader is referred to Refs. 35 and 42 for the rest of the related formulas for this propagator.

To compute real system properties connected to correlations when one starts from first principles and uses general interaction potential energies $U$, involving interactions beyond the pair level, the over-counting of effects must be avoided. The latter is clearly a task to be accomplished with atomic structure calculations, and computations can be performed with path integral techniques. Nevertheless, in the case of model systems, such as the composed of quantum hard spheres, to the knowledge of this author no special propagators even for triplet "collisions" have been obtained. On the other hand, for many purposes this absence may not be crucial, since pure triplet effects in this system are expected to play a significant role only at very high densities. ${ }^{6}$ This surmise finds support in the fact that quantum hard spheres repel each other before "classical contact" occurs.

Cao-Berne propagator is more efficient than the former "image" propagators, ${ }^{3,50}$ while being much easier to implement to carry out extensive computations than the more recent propagator proposed by de Prunelé. ${ }^{52}$ In addition, Cao-Berne propagator is expected to be sufficiently accurate for treating the magnitude of most of the quantum effects considered herein, as indicated by its extension of the results arising from the image propagators and the well-known semiclassical methods. ${ }^{63}$ Besides, as this article is mainly concerned with the methodological aspects of the $O Z 2$ direct calculation of isothermal compressibilities, the selection made can be regarded as most significant for these purposes.

PIMC simulations for the QHS fluid have employed the sample size $N_{S} \times P=500 \times 12$, except for those at $\lambda_{B}^{*}=2$ for which $N_{S} \times P=432 \times 24$. Both $P$ discretizations have been checked to give PIMC practical convergence. The particle mass and diameter have been set at the values $m=28.0134 \mathrm{amu}$ and $\sigma=3.5 \AA$. The algorithm employed has been that of the necklace normal modes, ${ }^{64}$ and the same procedure explained elsewhere has been followed. ${ }^{22(c)}$ For the stable fluid state points, initial fcc (or bcc for $N_{S}=432$ ) lattices have been employed to start their simulations. 
For the metastable fluid state points, their corresponding initial configurations have been set by rescaling the coordinates of the particles of a typical equilibrated configuration belonging to the state point at the immediately lower density. After equilibration the run lengths have been 800 kpasses for the state points within $0.2 \leq \lambda_{B}^{*} \leq 0.8$, and 1900 kpasses for the state points at $\lambda_{B}^{*}=2$ (1 kpass $=10^{3} N_{S} \times P$ attempted bead moves). In order to compute the subaverages each run has been subdivided in ten equal-lentgh blocks, i.e. $\alpha=1,2, \ldots, M_{B}=10$. The number of PIMC configurations analyzed for computing structures have been $8 \times 10^{4} \quad(P=12)$ and $1.6 \times 10^{5}$ $(P=24)$. These configurations have been selected at regular intervals along the run, the bin width being set to $\Delta r=0.1 \AA$ for the distance range scanned $L / 2$ (with the exceptions mentioned below for $\left.g_{E T}\right)$.

In evaluating the second derivative at "classical contact" in Eq. (30) there are always some elements of uncertainty as the density becomes higher, which are related to the width of the bins used to gather statistics near the hard core. In this work $g^{\prime \prime}{ }_{E T}(\sigma+)$ has been calculated through least squares fits by applying the behavior $g_{E T}(r)=a(r-\sigma)^{2}$ in the vicinity of $r=\sigma+{ }^{63(\mathrm{c})}$ The bins involved in these calculations of $g^{\prime \prime}{ }_{E T}(\sigma+)$ have been centered at $3.505,3.515$ and $3.525 \AA$ $\left[g_{E T}(\sigma) \equiv 0\right]$. The results obtained for the intervals $(3.5 \leq r / \AA \leq 3.515)$ and $(3.5 \leq r / \AA \leq \leq 3.525)$ have been combined via the weighting of averages, ${ }^{59}$ which smoothes the results and produces a lower variance.

Table I gives some salient results for the first peak regions of the ET and CM pair functions. The error bars reported are one-standard deviation, which has been obtained from the corresponding subaverages. These error bars remain lower than $0.2 \%(\mathrm{ET})$ and $0.6 \%(\mathrm{CM})$. For long distances $(\cong 15 \AA ̊)$ ) the error bars remain lower than $0.4 \%$ in all cases. Fierz's potential energy term in Eq. (30) has been obtained with a relative precision that diminishes with decreasing densities. For example, at $\lambda_{B}^{*}=2$ one finds for the Fierz's contribution to the compressibility factor $p V / R T$ the following results: a) $\rho_{N}^{*}=0.1,1.036 \pm 0.082 ;$ b) $\rho_{N}^{*}=0.2,4.042 \pm 0.140$; 
and c) $\rho_{N}^{*}=0.3,10.441 \pm 0.270$. With increasing densities this term becomes more and more important when calculating the virial pressure, as one can see by comparing the latter results with the following kinetic energy contributions to $p V / R T:$ a) $\rho_{N}^{*}=0.1,1.588 \pm 0.008 ;$ b) $\rho_{N}^{*}=0.2$, $2.967 \pm 0.010 \mathrm{c}) \rho_{N}^{*}=0.3,5.525 \pm 0.017$. (The errors quoted are one-standard deviation).

\section{B. BDH plus GC iterations}

$\mathrm{BDH}$ calculations for the pair necklace centroid correlations have been performed using two independent partitions in Eqs. (12), $n=220,440$, at equally spaced points within the selected range of distances, $\sigma \leq R / \AA \leq 15.5$. The number of trial distances $R_{T}$ (equally spaced) has been set to 65 . In practice, the latter partitions force one to work with kernels sized $(220 \times 220)$ and $(440 \times 440)$ when solving Eq. (12a). ${ }^{32,35,55}$ The conditions for convergence of the BDH merit function have been set to $F \leq 5 \times 10^{-6}$ or 300 iterations at the most, whichever occurs first. The latter condition guarantees $F \leq 10^{-4}$ for most of the cases investigated, which is known to yield sufficiently accurate results in Fourier space. More details of these calculations can be found in Appendix IV.

The mid-upper range of significant distances for the $R_{Z}$ zeros to appear has been set to $8.5 \leq R / \AA \leq 15.5$, which has been found to serve as a common range for all the state points investigated. The implicit finite-size effects have been neglected and the GC iterations have been carried out up to $m_{\max }=5$, which has produced a precision $\leq 10^{-7}$ for the individual estimates $S_{C M}(k=0)$ at each $R_{Z}$. To grasp the reliability of these selections Table II contains the results for the $S_{C M}(0)$ values at $\left(\lambda_{B}^{*}=0.6, \rho_{N}^{*}=0.5\right)$. Typical behaviors at the stable and metastable fluid state points can be summarized by saying: i) the GC iterations generally increase the number of $R_{Z}$ zeros, with respect to the initial $\mathrm{BDH}(m=0)$ results, an effect more noticeable for lower densities; ii) the first GC step produces most of the correction to $S(k=0)$; and iii) there is a close clustering of the dimensionless compressibilities about a mean value $\left\langle S_{C M}(0)\right\rangle$. 
It is worth stressing that a single $\mathrm{BDH}+\mathrm{GC}$ application is a very fast task in computational terms. Roughly speaking, on a modern-day average computer it may take a time in between seconds (low- $\rho_{N}^{*}$ and low- $\lambda_{B}^{*}$ state points) and some hours. (For state points deep inside the metastablity regime it may take one day). It is then a low-cost operation with a wide range of answers and potential applications through the knowledge of $c(r){ }^{46}$

\section{RESULTS}

\section{A. Isothermal compressibilities}

The general trends shown by the two $n$-partitions employed have been found similar. The quantitative differences between both numerical conditions are generally small, although they become noticeable at the highest densities, where the $(440 \times 440)$ results become more accurate. Table III contains representative final results obtained for the isothermal compressibility along the five isotherms. It is worthwhile to remark that in using $\mathrm{BDH}(m=0)$ the very existence of $R_{Z}$ zeros for the $\left\langle h_{C M}\left(R_{12}\right)\right\rangle$ centroid correlations is a numerical fact in all the cases investigated. With the exception of state point $\left(\lambda_{B}^{*}=2, \rho_{N}^{*}=0.1\right)$, for which the condition $\left[Q^{\prime}\left(R_{Z}\right) / R_{Z}\right]^{2}=$ minimum has been needed, the number of $R_{Z}$ zeros arising from the $m=0$ calculations have always been greater than one. One might expect that a thorough sampling would influence this BDH feature, for instance, by reducing the number of zeros. Roughly speaking, such an effect is observed in the current calculations when the results arising from "mean" and from "blocks" are considered, but not to the extent of making this number close to unity. This is also to be compared with Ref. 36, where the sampling was much less demanding $\left(N_{S}=125, P=3,6\right.$, and 54 kpasses $)$ and more than one $R_{Z}$ was obtained for $\rho_{N}^{*} \geq 0.3$.

The GC iterations produce, in most of the state points, more zeros than the only application

of BDH at step $m=0$. Therefore, one finds zeros within the significant range, $8.5 \leq R / \AA \leq 15.5$, which would otherwise be either spoilt or hidden by the finite- $N$ effects. Besides, this behavior 
lends support to the approach proposed in this article to evaluate errors in the isothermal compressibility [Eq. (23)]. It also indicates that, in running grand ensemble simulations, a number of zeros significantly greater than unity would be obtained via BDH. Only reference to the zeros contained in that mid-upper range of distances, or to the largest one if $R_{Z}<8.5 \AA$, is made in Table III. It is interesting to highlight that $S_{C M}(k=0)$ diminishes as $\lambda_{B}^{*}$ increases, which agrees with the expected theoretical trend $S(k=0) \rightarrow 0$ as $T \rightarrow 0 .{ }^{60}$

Putting aside just a few exceptions at low densities and de Broglie wavelengths, GC corrections increase the isothermal compressibility obtained at stage $\operatorname{BDH}(m=0)$, an effect that turns out to be more important with the quantum effects. For example, the magnitude of the GC corrections is: a) $0.3 \%$ at $\left(\lambda_{B}^{*}=0.2, \rho_{N}^{*}=0.1\right)$, and $6 \%$ at $\left(\lambda_{B}^{*}=0.2, \rho_{N}^{*}=0.805\right) ;$ b) $1 \%$ at $\left(\lambda_{B}^{*}=0.8, \rho_{N}^{*}=0.1\right)$, and $3.5 \%$ at $\left(\lambda_{B}^{*}=0.8, \rho_{N}^{*}=0.545\right) ;$ and $\left.\mathrm{c}\right) 1 \%$ at $\left(\lambda_{B}^{*}=2, \rho_{N}^{*}=0.1\right)$, and $2.7 \%$ at $\left(\lambda_{B}^{*}=2, \rho_{N}^{*}=0.2\right)$. The pattern shown by the results arising from the GC applications to the groups of ten block structures $\bar{h}_{C M, \alpha}\left(R_{12}\right)$ is analogous. This cumulative increasing in $\chi_{T}$ will lower the final fluid pressure computed with Eq. (11).

On the other hand, there is a close proximity between $\left\langle\chi_{T}\right\rangle$ and $\bar{\chi}_{T}$. Also, along the whole series of calculations one finds the expected behavior: $s^{2}\left(\left\langle\chi_{T}\right\rangle\right) /\left(s^{2}\left(\bar{\chi}_{T}\right) \sum_{\alpha} K^{G C}(\alpha)\right)<1$. For example, at $\rho_{N}^{*}=0.5(440 \times 440)$ that quotient is: a $) \approx 0.03$ at $\lambda_{B}^{*}=0.2 ;$ b) $\approx 0.08$ at $\lambda_{B}^{*}=0.4 ;$ c $)$ $\approx 0.1$ at $\lambda_{B}^{*}=0.6 ;$ d) $\approx 0.6$ at $\lambda_{B}^{*}=0.8 ;$ and $\left.\mathrm{e}\right) \approx 0.4$ at $\left(\rho_{N}^{*}=0.2, \lambda_{B}^{*}=2\right)$. It is worth remarking that this behavior is systematic, except for state point $\left(\rho_{N}^{*}=0.3, \lambda_{B}^{*}=2\right)$ where the quotient is $\approx 1$

As regards the statistical distribution of the $\chi_{T}$ values, every entire set $\left\{\chi_{T, \eta(\alpha)}\right\}_{\alpha=1, M_{B}}$ for each state point obtained in this work has been analyzed for consistency with the Gaussian and the log-normal densities. To this end, use of the Kolmogorov-Smirnov test has been made. ${ }^{65}$ Although the samples are large (in between 65 and 184 elements), the obtained results show rather diverse 
patterns and do not lead to any general conclusive identification of a normal population. Therefore, the complementary comparison at each state point investigated between $s\left(\left\langle\chi_{T}\right\rangle\right)$ and each individual block dispersion $s\left(\chi_{T, \eta(\alpha)}\right), \alpha=1,2, \ldots, 10$, has been made by using the sign test. In so doing, deviations from the behavior $s\left(\left\langle\chi_{T}\right\rangle\right)<s\left(\chi_{T, \eta(\alpha)}\right)$ have been sought for.

Out of the ten cases per state point one finds along each isotherm the following behaviors. Along $\lambda_{B}^{*}=0.2$ no deviations are detected for most of the state points, and at some of them at high densities only one deviation appears. Along $\lambda_{B}^{*}=0.4$ the same general result is obtained, although at some state points at high densities one or two deviations appear. Along $\lambda_{B}^{*}=0.6$ and $\lambda_{B}^{*}=0.8$ the number of deviations is $\leq 2$, with the exception of $\left(\lambda_{B}^{*}=0.8, \rho_{N}^{*}=0.5\right)$ where 3 deviations appear. Along $\lambda_{B}^{*}=2$ the number of deviations is 3 at $\rho_{N}^{*}=0.1$ and 0.2 , and 5 at $\rho_{N}^{*}=0.3$. Application of the robust sign test indicates that for $\lambda_{B}^{*} \leq 0.8$, and with probability $\approx 95 \%$, one cannot reject (may accept) that $s\left(\left\langle\chi_{T}\right\rangle\right)<s\left(\chi_{T, \eta(\alpha)}\right)$ for the observed behavior, since the probability of random appearance of two negative deviations, at the most, is $\approx 0.055$ (i.e. usual critical level $=0.05)$. At $\left(\lambda_{B}^{*}=0.8, \rho_{N}^{*}=0.5\right)$ and at $\left(\lambda_{B}^{*}=2, \rho_{N}^{*}=0.1,0.2\right)$ the situation may be regarded as reasonably satisfactory, since the probability of not rejecting the proposed behavior turns out to be $\approx 83 \%$. The extreme case appearing at state point $\left(\lambda_{B}^{*}=2, \rho_{N}^{*}=0.3\right)$ presents a probability of no rejection that dramatically diminishes to $\approx 38 \%$, which is in accord with the abovementioned ratio of variances $\approx 1$.

Putting the foregoing results together one concludes that, for the overwhelming majority of the present results (44 state points out of 45), the analysis performed gives significant, or at least valuable, answers regarding the error bars in the $\chi_{T}$ computed. The final error bars $\varepsilon\left(\left\langle\chi_{T}\right\rangle\right)$ Eq. (23) remain very well controlled (Table III) and their relative importance to the estimates $\left\langle\chi_{T}\right\rangle$ can be summarized as follows. First, for the stable fluid state points the $\varepsilon\left(\left\langle\chi_{T}\right\rangle\right)$ values stay generally 
below $1-2 \%$. Second, the $\varepsilon\left(\left\langle\chi_{T}\right\rangle\right)$ values show a rise with the density as the fluid conditions approach and enter the metastable regions, but they remain lower than $4.6 \%$, which is the highest deviation obtained $\left(\lambda_{B}^{*}=0.4, \rho_{N}^{*}=0.695\right)$. In this connection, small departures from purely monotonic behaviors are also observed as the density increases. Clearly, as the quantum effects increase, larger block sizes are needed to improve further the accuracy in this sort of calculations.

\section{B. Equations of state}

Table IV contains representative results for different versions of the fluid equation of state (see Ref. 61 for full data). These results are to be compared between them and with those reported in Refs. 28, 30(a) and 36, where the sample sizes employed were $\left(N_{S} \times P=500 \times 12\right)$ and $\left(N_{S}=125, P=3,6\right)$, and the gathering of statistics was far from being so complete. Errors (onestandard deviation) in the energies and virial pressures have been fixed with the corresponding sub-averages.

For $\lambda_{B}^{*} \leq 0.8$ there is a good agreement for the mean total internal energies computed herein and those previously reported, although the present error bars are significantly smaller. Four compressibility factors $P V / R T$ are shown: the one obtained through the virial formula Eq. (30), those obtained through $\mathrm{BDH}+\mathrm{GC}(m=5)$ with the two kernels employed, and that obtained through only the initial BDH application $[G C(m=0)]$ with the largest kernel. These results also show an excellent overall agreement between the four $P V / R T$, albeit a number of significant details have to be mentioned. Firstly, the $\mathrm{GC}(m=5)$ corrections diminish $P V / R T$ and are more important with the density, reaching $\approx-4 \%$ with respect to $m=0$ for some metastable fluid state points. Secondly, for the stable fluid state points there is little influence of the BDH kernel size on the error bars and also on the $P V / R T$ mean values. In this connection, one observes that if the kernel is enlarged, slightly better accuracy is obtained when the metastable regime at the lowest temperatures $\left(\lambda_{B}^{*}=0.8,2\right)$ is approached. Thirdly, by increasing the density along every isotherm one observes that the BDH-GC $(440 \times 440)$ calculations begin to yield much smaller error bars than 
those of the virial estimates, but this trend may become less pronounced, or even reversed, at some high density metastable fluid state points.

The isothermal compressibilities that can be calculated from the $P V / R T$ virial values agree reasonably well with those of $\mathrm{BDH}+\mathrm{GC}(m=5)$, although their error bars turn out to be larger. For example, using numerical differentiation (e.g. Stirling's, Richardson's extrapolation) for the virial data, the values $\chi_{T} / 10^{-3} b a r^{-1}$ obtained are: a) at $\left(\lambda_{B}^{*}=0.8, \rho_{N}^{*}=0.53\right)$ virial= $7.831 \pm 0.989, \quad \mathrm{BDH}+\mathrm{GC}=7.452 \pm 0.207 ; \quad$ b $)$ at $\left(\lambda_{B}^{*}=0.8, \rho_{N}^{*}=0.3\right) \quad$ virial $=67.560 \pm 2.590$, $\mathrm{BDH}+\mathrm{GC}=68.388 \pm 0.317$; and $\mathrm{c})$ at $\left(\lambda_{B}^{*}=2, \rho_{N}^{*}=0.2\right)$ virial $=343.611 \pm 8.898, \mathrm{BDH}+\mathrm{GC}=$ $354.038 \pm 4.977$.

As regards the comparison of the $P V / R T$ values based on $\mathrm{BDH}+\mathrm{GC}$ with those obtained in previous $\mathrm{CM}-\mathrm{BDH}(m=0)$ calculations, ${ }^{28,30(\mathrm{a}), 36}$ the current ones appear systematically lower because of the higher isothermal compressibilities obtained. In this regard, the discrepancies are more important with increasing densities as indicated by the following results: ${ }^{61}$ a) $\approx-8 \%$ at $\left(\lambda_{B}^{*}=0.2, \rho_{N}^{*}=0.775\right) ;$ and $\left.\mathrm{b}\right) \approx-2 \%$ at $\left(\lambda_{B}^{*}=0.8, \rho_{N}^{*}=0.530\right)$. Therefore, some repercussions on the fluid-solid equilibria of this system, as reported in Ref. 30(a), may then be expected from the current fluid results.

\section{Structural results}

Finally, and for further reference, Figs. 1 to 3 show some structural features at state point $\left(\lambda_{B}^{*}=2, \rho_{N}^{*}=0.2\right)$ with the kernel $(440 \times 440)$.

Fig. 1 illustrates the convergence attained in the isothermal compressibility by displaying the close proximity between the centroid direct correlation functions obtained. Only the $c_{C M}(R)$ at $R_{Z}=10.056 \AA$ and at $R_{Z}=14.315 \AA$ are shown, since the intermediate results evolve in a very smooth fashion and the details cannot be seen on the scale of the graph. Fig.2 shows the pair radial correlation functions (CM, ET and PLR) and, at this point, it is worthwhile to dwell a little on the differences between these three types of radial functions. 
Firstly, the CM-ET/PLR comparison reveals a much more structured function for the centroids, which can be understood by noting that they "mimic" a fluid at a higher density than the actual one, as every centroid is embedded into a "shell" of hard beads. This situation is somewhat similar to that occurring in some molecular fluids. For example, in liquid $\mathrm{CH}_{4}$ the intermolecular radial function $g(C-C)$-the "centroid" function- shows higher peaks and larger oscillations than $g(H-H)$-the "PLR" function-. ${ }^{66}$ In a sense, the CM function is "more" classical than the ET or the PLR functions, ${ }^{31,47}$ which display fully the well-known quantum smearing out of the classical features (diminished peaks, raised valleys, and rightward shift). Moreover, note that the CM function incorporates quantum effects, which can be checked by comparison with the corresponding classical radial function that: a) may show its features shifted inwards with respect to $\mathrm{CM}$ (e.g. hard-sphere fluid), ${ }^{25}$ or b) can be even more structured than CM (e.g. liquid parahydrogen, ${ }^{47}$ gaseous helium-3). ${ }^{41}$

Secondly, the ET-PLR comparison shows that ET vanishes at $r=\sigma+$, as no tunneling is possible, whereas PLR appears to be "penetrating" inside the hard core. The latter fact is related to: a) the linear response from the fluid to a continuous weak external field; ${ }^{25,28}$ and b) sum rules involving the dynamic structure factor. ${ }^{48,60}$ Although PLR is more "smeared out" than ET, because the former involves more centers for the average, one has to be aware that in the PLR case no actual invasion of the hard core takes place. This PLR feature is due to the fact that the system correlates with itself at different imaginary time slices, and the reader is referred to Ref. 4 for an early and insightful discussion of this issue.

Thirdly, given the asymptotic behavior $g(r) \rightarrow 1$, the differences CM-ET-PLR will greatly diminish for long distances. However, their exact decay properties are expected to be different, as suggested by the QHS dominant pole analysis reported in Ref. 39. These three functions should show exponentially oscillatory decay, but the period and inverse decay length of the CM oscillations should be smaller than those of the ET and the PLR cases. 
Fig. 3 shows the associated k-space response functions. It has been assumed that $O Z 2$ is also a valid equation for the ET and the PLR correlations. Although on the scale of the graph the (ET/PLR) - CM discrepancies between the $S(k=0)$ values are small, they actually amount to $+35 \%(\mathrm{ET})$ and $+31 \%(\mathrm{TLR})$ taking $S_{C M}(k=0)=0.05063$ as a reference. The analogous results obtained at $\left(\lambda_{B}^{*}=2, \rho_{N}^{*}=0.1\right)$ show a dramatically different behavior, since they are quite close to each other (discrepancies $<+3 \%$ ). Therefore, the foregoing behavior at $\left(\lambda_{B}^{*}=2, \rho_{N}^{*}=0.2\right)$ indicates the inadequacy of $O Z 2$ for fixing accurate $\chi_{T}$ values from the ET or the PLR correlations under such strong diffraction conditions. However, past the low- $k$ region the latter estimates of the ET and TLR structure factors at $\rho_{N}^{*}=0.2$ are still expected to capture most of the salient features of these Fourier space functions. ${ }^{27,29,41}$ Fig.3 also shows the TLR function resulting from approximating the self correlations $s_{S C}(r)$ with an analytical approach based on the FeynmanHibbs picture, ${ }^{32}$ which gives quite a reasonable result in comparison with $\mathrm{PI}-\mathrm{P}=24$. Further improvements in accuracy for the ET and TLR structure factors could be achieved by using

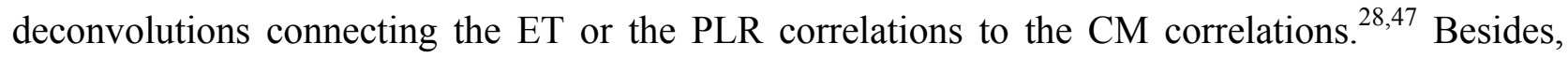
efficient descriptions of the self correlations $s_{S C}(r)$ are needed, ${ }^{21}$ because the $P$ convergence involved turns out to be rather slow, ${ }^{25,41}$ and the analytical approach mentioned ${ }^{32}$ cannot cope with increasing quantum dispersion effects.

\section{CONCLUSION}

The fact that the present work is based on fundamental classical methodology is very rewarding in that it is in the spirit of the PI classical isomorphism. ${ }^{4}$ As shown, the PI centroids are a most useful device for counting number fluctuations via the $O Z 2$ equation. The $\mathrm{BDH}+\mathrm{GC}$ procedure employed appears as highly satisfactory in that: a) it has a broad scope, since it is connected to the wide range of problems associated with the direct correlation functions in the real and in the Fourier spaces; ${ }^{46}$ and $\mathrm{b}$ ) the $\chi_{T}$ estimates corrected for finite- $N$ effects can be 
determined in a very fast computational way. $\mathrm{BDH}+\mathrm{GC}$ improves greatly the long distance behavior of the centroid pair radial correlation functions obtained with canonical PIMC simulations. As a result, application of this procedure reveals the significant $R_{Z}$ zeros that define the isothermal compressibility, which otherwise would be either spoilt or hidden by the finite- $N$ effects. The fact that these zeros exist when properly dealing with the BDH method may be regarded as established numerically, and one can expect the same result from the grand-canonical simulation+BDH approach to the problem. All of this lends support to the use of Eq. (23) for obtaining the error bars in the mean isothermal compressibility determined via $\mathrm{BDH}+\mathrm{GC}$. Although Eq. (23) is expected on physical grounds to be a consistent estimator, i.e. one that tends to zero by enhancing statistics, this is a question that boils down to the role played by the intrinsic dispersion $s^{2}\left(\left\langle\chi_{T}\right\rangle\right)$. Although this question could only be settled by extensive calculations, the current results have proven that all the contributions behave properly. Therefore Eq. (23) can be safely regarded as both significant and useful, at least for most practical purposes in quantum and classical applications.

The GC corrections increase the QHS isothermal compressibilities that would be obtained by simply using the BDH method only once. Most of the magnitude of these corrections is achieved in the first step of the GC procedure. Accordingly, the QHS compressibility factors arising from $\mathrm{BDH}+\mathrm{GC}$ are lower than those of the $\mathrm{BDH}$ method. Also, it is interesting to point out that the virial equation of state obtained through Eq. (30) is in good accord with the equation of state arising from the integration of $\chi_{T}$ Eq. (11). This result is reassuring regarding the suitability of the method followed to determine the second derivative of the instantaneous function at classical contact. One might think of a very detailed simulation scheme for extracting a precise measure of the curvature of the ET function at the hard core, but for a given accuracy the $\chi_{T}$ centroid route turns out to be much faster in computational terms. In view of the particular characteristics of the quantum hard sphere interactions, it is expected that most of the finite- $N$ effects in the current canonical calculations $\left(N_{S}=500\right)$ have been corrected with $\mathrm{BDH}+\mathrm{GC}$. 
Again, this is indicated by the closeness between the virial and the $\chi_{T}$ equations of state obtained. Within the canonical framework, for stable as well as metastable fluid state points, the computation of pressures based on $\chi_{T}$ should be the preferred ones, as they present less elements of uncertainty than those linked to the evaluation of the ET second derivative in the virial pressure.

The $\chi_{T}$ route is expected to become valuable when studying fluid-solid coexistence properties at large $\lambda_{B}^{*}$ values via the calculation of fluid Helmholtz free energies. ${ }^{30(a)}$ The current results have been obtained through a much improved gathering of statistics and indicate lower isothermal compressibilities than those reported in Ref. 30 (a). Therefore, a number of differences with respect to Ref. 30(a) are to be expected in the fluid-solid coexistence properties of the quantum hard-sphere system. Since in the experimental domain the fluid-solid equilibrium for classical hard spheres was stated by Pusey and van Megen to agree with early simulation results, ${ }^{67}$ it is hoped that computational results on quantum hard spheres may stimulate experimental research on systems composed of colloidal particles at very low temperatures.

\section{ACKNOWLEDGMENT}

Financial support from Comunidad de Madrid (Spain) through Project MODELICO (P2009/ESP$1691)$ is gratefully acknowledged.

\section{APPENDIX I}

The centroid density fluctuations in Fourier space, under the particular action of the nonlocalizing weak external field of constant strength, are obtained by taking the Fourier transform in Eq. (7b) and by using the approximations $\rho_{N, C M}^{(2)}\left(\Psi_{F}\right) \approx \rho_{N}^{2} g_{C M}(R)$ and $\rho_{N, C M}^{(1)}\left(\Psi_{F}\right) \approx \rho_{N}$. One finds then $\delta \rho_{N, C M}^{(1)}\left(\mathbf{k}, \Psi_{F}\right)=-\beta \rho_{N} S_{C M}(\mathbf{k}) \delta \Psi_{F}(\mathbf{k})$

where $S_{C M}(k)$ is the CM structure factor Eq. (8). The OZ2 equation in the presence of $\Psi_{F}$ arises from the usual definition of the direct correlation function ${ }^{46}$ 
$c_{C M}\left(\mathbf{R}_{1}, \mathbf{R}_{2} ; \Psi_{F}\right)=\frac{\delta\left(\mathbf{R}_{1}-\mathbf{R}_{2}\right)}{\rho_{N, C M}^{(1)}\left(\mathbf{R}_{1} ; \Psi_{F}\right)}+\frac{1}{k_{B} T} \frac{\delta \Psi_{F}\left(\mathbf{R}_{2}\right)}{\delta \rho_{N, C M}^{(1)}\left(\mathbf{R}_{1} ; \Psi_{F}\right)}$

and the application of the functional identity

$$
\int d \mathbf{R}_{3} \frac{\delta \rho_{N, C M}^{(1)}\left(\mathbf{R}_{1} ; \Psi_{F}\right)}{\delta \Psi_{F}\left(\mathbf{R}_{3}\right)} \times \frac{\delta \Psi_{F}\left(\mathbf{R}_{3}\right)}{\delta \rho_{N, C M}^{(1)}\left(\mathbf{R}_{2} ; \Psi_{F}\right)}=\delta\left(\mathbf{R}_{1}-\mathbf{R}_{2}\right)
$$

The OZ2 equation for the homogeneous fluid Eq. (9) is obtained by setting $\Psi_{F}=0$ in the expression arising from (A1.3). Note that the functional derivatives involved do have a consistent meaning in the grand ensemble, since the density is a fluctuating quantity.

\section{APPENDIX II}

Enter $\left\langle h_{C M}\left(R_{12}\right)\right\rangle$ into the procedure Eqs. (12) to (17). The set of possible solutions $\left\{R_{Z}, c\left(r ; R_{Z}\right), S\left(k ; R_{Z}\right)\right\}_{B D H}$ arising from $\mathrm{BDH}(m=0)$ serves to obtain a first estimate of the physically significant (mid-upper) range of distances where the $R_{Z}$ zeros are to be found. Normally, this mid-upper range remains unaltered throughout the GC steps $m \geq 1$, and it is easily recognizable by the slight fluctuations of $\chi_{T}$ about a mean value. Once this range and the set of solutions have been refined via Eqs. (17), one obtains the final set $\left\{R_{Z}, c\left(r ; R_{Z}\right), S\left(k ; R_{Z}\right)\right\}_{B D H+G C}$ and the final mid-upper range of zeros $R_{Z 1} \leq R_{Z, \nu} \leq R_{Z K^{G C}}$. At a given step $m$ the value $S_{C M}^{(m-1)}(0)$ is determined as the average of the $S_{C M}(k=0)$ values appearing within the $(m-1)^{\text {th }}$ mid-upper range of distances. After the $\mathrm{BDH}+\mathrm{GC}$ iterations have been completed, one calculates the mean isothermal compressibility of the whole run as the final average Eq. (19), and a measure of its precision is Eq. (20). The latter quantity is likely to be higher than the actual error for the mean, but in view of the relatively low value of $K^{G C}(<10)$ it is a safe option.

The short and long ranges of $R_{Z}$ zeros, namely $\sigma<R_{Z}<R_{Z 1}$ and $R_{Z K^{G C}}<R_{Z}<L / 2$, are to be discarded. The former because they are strongly influenced by the interatomic repulsions, which make $\chi_{T}$ behave far from the stable way mentioned above. The latter because they can be affected by the finite- $N$ effects that might still remain uncorrected by GC, a fact that may bring about 
sudden changes in $\chi_{T}$ with respect to the stable mean value $\left\langle\chi_{T}\right\rangle$ attained. The final basic range $R_{Z 1} \leq R_{Z, v(\alpha)} \leq R_{Z K^{G C}}$ will serve to set the significant mid-upper range of distances for the zeros, and thus to fix their related parameters [i.e. $\left.\left\langle S_{C M}^{(m-1)}(0)\right\rangle\right]$ for carrying out the complementary error analysis of the blocks that follows.

For a sufficiently long block length it is expected that the application of the same complete procedure to every sub-average PI centroid structure $\bar{h}_{C M, \alpha} \quad\left(\alpha=1,2, \ldots, M_{B}\right)$ gives a good approximation $\bar{\chi}_{T, \alpha}$ to $\left\langle\chi_{T}\right\rangle$. One computes the average over the $R_{Z, \eta(\alpha)}$ zeros found $\left(K^{G C}(\alpha)\right.$ in number) within the basic range and finds the estimates per block $\alpha$

$$
\begin{aligned}
& \bar{\chi}_{T, \alpha}=\frac{1}{K^{G C}(\alpha)} \sum_{\eta(\alpha)=1}^{K^{G C}(\alpha)} \chi_{T, \eta(\alpha)} \\
& s^{2}\left(\chi_{T, \eta(\alpha)}\right)=\frac{1}{K^{G C}(\alpha)-1} \sum_{\eta(\alpha)=1}^{K^{G C}(\alpha)}\left(\chi_{T, \eta(\alpha)}-\bar{\chi}_{T, \alpha}\right)^{2}
\end{aligned}
$$

By collecting the results obtained in all the blocks one computes the "pooled" quantities" Eqs. (21) and (22). Now, given that the whole amount of zeros is a large number, the use of Eq. (22) makes sense. With the above information $\varepsilon\left(\left\langle\chi_{T}\right\rangle\right)$ can be fixed via Eq. (23).

\section{APPENDIX III}

The main properties of the estimator Eq. (23) may be understood in connection with the basic integral equation of the whole process Eq. (12a). The situation can be summarized in the following basic integral equations for the necklace centroid correlations $\left(R_{12}>0\right)$

$$
\begin{aligned}
& R_{12}\left\langle h_{C M}\left(R_{12}\right)\right\rangle=-\left\langle Q^{\prime}\left(R_{12}\right)\right\rangle+2 \pi \rho_{N} \int_{0}^{R} d r\left(R_{12}-r\right)\left\langle h_{C M}\left(\left|R_{12}-r\right|\right)\right\rangle\langle Q(r)\rangle \\
& R_{12} \bar{h}_{C M, \alpha}\left(R_{12}\right)=-\bar{Q}_{\alpha}^{\prime}\left(R_{12}\right)+2 \pi \rho_{N} \int_{0}^{R} d r\left(R_{12}-r\right) \bar{h}_{C M, \alpha}\left(\left|R_{12}-r\right|\right) \bar{Q}_{\alpha}(r) ; \quad \alpha=1,2, \ldots, M_{B} \\
& R_{12} \frac{1}{M_{B}} \sum_{\alpha=1}^{M_{B}} \bar{h}_{C M, \alpha}\left(R_{12}\right)=-\frac{1}{M_{B}} \sum_{\alpha=1}^{M_{B}} \bar{Q}_{\alpha}^{\prime}\left(R_{12}\right)+2 \pi \rho_{N} \frac{1}{M_{B}} \sum_{\alpha=1}^{M_{B}} \int_{0}^{R} d r\left(R_{12}-r\right) \bar{h}_{C M, \alpha}\left(\left|R_{12}-r\right|\right) \bar{Q}_{\alpha}(r) ;
\end{aligned}
$$


where a common upper limit $R$ for the integrations has been taken. Eq. (A3.3) results from the summation and division by $N_{B}$ of the set of equations (A3.2) and it cannot be made formally equivalent to Eq. (A3.1). It is clear on physical grounds, however, that as one makes the sub-length of the blocks larger, the differences between any of the $\bar{h}_{C M, \alpha}$ and $\left\langle h_{C M}\left(R_{12}\right)\right\rangle$ may be made as small as desired. Accordingly, one should find that for sufficiently large $\alpha$-block sizes

$$
\left\langle Q^{\prime}\left(R_{12}\right)\right\rangle \approx \bar{Q}_{\alpha}^{\prime}\left(R_{12}\right) \approx \frac{1}{M_{B}} \sum_{\alpha=1}^{M_{B}} \bar{Q}_{\alpha}^{\prime}\left(R_{12}\right)
$$

Then, by applying Eq. (14b) one would obtain

$$
\langle S(k=0)\rangle=\{\langle Q(k=0)\rangle\}^{-2} \approx \bar{S}_{\alpha}(k=0)=\left\{\bar{Q}_{\alpha}(k=0)\right\}^{-2} \approx \bar{S}(k=0)=\left\{\frac{1}{M_{B}} \sum_{\alpha=1}^{M_{B}} \bar{Q}_{\alpha}(k=0)\right\}^{-2}
$$

By enhancing statistics the sets of solutions $\left\{R_{Z}, Q^{\prime}\left(r ; R_{Z}\right)\right\}_{B D H}$ corresponding to these three versions of the PI centroid problem would turn out to be almost indistinguishable from each other. Therefore $\left|\left\langle\chi_{T}\right\rangle-\bar{\chi}_{T}\right|$, as defined by Eqs. (19) and (21), may be made as small as desired. A more detailed discussion including the role of the different zeros $R_{Z v}$ obtained for each of the three cases leads to the same general result, since $Q$ and $Q^{\prime}$ can be extended with zeros beyond their respective $R_{Z}$ ranges of definition. ${ }^{54,55}$ Alternatively, the situation may be more easily visualized by considering in Eq. (14b) the role played by the consecutive approaches to the direct correlation function. The latter will be very close to one another within the common range of distances, while beyond that range the magnitude of their differences will be truly small, in an analogous fashion to that shown in Fig. 1. As regards the contribution $s^{2}\left(\bar{\chi}_{T}\right)$, it can also be made as small as desired by increasing both the block sub-length and the number of blocks. With these operations the definition of the significant zeros is expected to be more and more precise, and thereby that of $\left\langle\chi_{T}\right\rangle$. All of this, in the limiting case of a very long run, leaves $s^{2}\left(\left\langle\chi_{T}\right\rangle\right)$ as the only source of statistical error in $\left\langle\chi_{T}\right\rangle$. 


\section{APPENDIX IV}

The solving of the linear system Eq. (16) has been carried out by using LU decomposition augmented with the iterative improvement of the solution (tolerance $10^{-10}$ ) as described in Ref. 65. To obtain the solution of Eqs. (12) the numerical integrations have been carried out by employing a natural cubic spline algorithm (optimal quadrature). The use of the two partitions mentioned in the main text allows one to assess the final precision reached in the compressibility factor. The numerical integrations to determine Fourier space properties have been carried out with Filon quadrature ${ }^{43}$ by using 2999 equally spaced points, which have also been obtained with natural cubic splines [final precision in $S(k=0) \sim 10^{-9}-10^{-10}$ ].

The $F$ minimizations have converged properly, even when analyzing the metastable fluid state points. Application of Eq. (14a) has not been needed to discard $R_{Z}$ zeros in the "mean" calculations, albeit the application of this criterion has been necessary in the analysis of a few block structures $\bar{h}_{C M, \alpha}\left(R_{12}\right)$. Although in most of the calculations $F<10^{-6}$ or $10^{-5}$, in a few metastable cases (at the highest densities for every isotherm) there have appeared $R_{Z}$ zeros, for which $F \sim 10^{-3}$, located in the middle region of the mid-upper range of distances. This feature still persists when increasing the number of BDH iterations $(>300)$. There are thus some special $r$ regions where the $F$ minimization cannot be forced below $\sim 10^{-3}$. In spite of this, the isothermal compressibilities associated with this sort of zeros agree very well with the trend shown by the others. Besides, past these regions the convergences for the rest of the larger zeros fall again within the standard F- margins given above. Finally, the integration of the compressibility equation Eq. (11) has also been carried out with natural cubic splines 


\section{References}

${ }^{1}$ R. P. Feynman and A. R. Hibbs, Quantum Mechanics and Path-Integrals (McGraw-Hill, New York, 1965).

${ }^{2}$ R. P. Feynman, Statistical Mechanics (Benjamin, Reading, 1972).

${ }^{3}$ J. A. Barker, J. Chem. Phys. 70, 2914 (1979).

${ }^{4}$ D. Chandler and P. G. Wolynes, J. Chem. Phys. 74, 4078 (1981).

${ }^{5}$ B. J. Berne and D. Thirumalai, Annu. Rev. Phys. Chem. 37, 401 (1986).

${ }^{6}$ D. M. Ceperley, Rev. Mod. Phys. 67, 279 (1995).

${ }^{7}$ L. M. Sesé, Mol. Phys. 81, 1297 (1994); 85, 931 (1995).

${ }^{8}$ J. Doll, D. L. Freeman and T. L. Beck, Adv. Chem. Phys. 78, 61 (1990).

${ }^{9}$ M. Müser and B. J. Berne, Phys. Rev. Lett. 77, 2638 (1996); D. Marx and M. H. Müser, J. Phys. Condensed Matter 11, R117 (1999).

${ }^{10}$ M. Suzuki, in Computer Simulation Studies in Condensed Matter Physics VIII, edited by D. P. Landau, K. K. Mon and H. B. Schüttler (Springer-Verlag, New York, 1996); S. A. Chin and C. R. Chen, J. Chem. Phys. 114, 7338 (2001); R. E. Zillich, J. M. Mayrhofer and S. A. Chin, J. Chem. Phys. 132, 044103 (2010).

${ }^{11}$ S. Jang, S. Jang and G. A. Voth, J. Chem. Phys. 115, 7832 (2001)

${ }^{12}$ K. Suzuki, M. Tachikawa and M. Shiga, J. Chem. Phys. 132, 144108 (2010)

${ }^{13}$ R. A. Kuharski and P. J. Rossky, J. Chem. Phys. 82, 5164 (1985); E. G. Noya, L. M. Sesé, R. Ramírez, C. McBride, M. M. Conde and C. Vega, Mol. Phys. 109, 149 (2011).

${ }^{14}$ M. Parrinello and A. Rahman, J. Chem. Phys. 80, 860 (1984).

${ }^{15}$ (a) M. E. Tuckerman, B. J. Berne, G. J. Martyna and M. L. Klein, J. Chem. Phys.99,2796(1993);

(b) D. Scharff, G. J. Martyna and M. L. Klein, J. Chem. Phys. 99, 8997 (1993); (c) G. J. Martyna, A. Hughes and M. E. Tuckerman, J. Chem. Phys. 110, 3275 (1999); (d) E. Balog, A. L. Hughes and G. Martyna, J. Chem. Phys. 112, 870 (2000).

${ }^{16}$ J. Cao and G. A. Voth, J. Chem. Phys. 100, 5093 (1994); 100, 5106 (1994); 104, 273 (1996).

${ }^{17}$ M. E. Tuckerman, D. Marx, M. L. Klein and M. Parrinello, J. Chem. Phys. 104, 5579 (1996). 
${ }^{18}$ M. Benoit, D. Marx and M. Parrinello, Nature (London) 392, 258 (1998).

${ }^{19}$ (a) R. Ramírez, T. López-Ciudad and J. C. Noya, Phys. Rev. Lett. 81, 3303 (1998); (b) R.

Ramírez and T. López-Ciudad, J. Chem. Phys. 111, 3339 (1999).

${ }^{20}$ I. R. Craig and D. E. Manolopoulos, J. Chem. Phys. 121, 3368 (2004).

${ }^{21}$ L. Lin, J. A. Morrone, R. Car and M. Parrinello, Phys. Rev. Lett. 105, 110602 (2010).

22 (a) R. P. Feynman and H. Kleinert, Phys. Rev. A 34, 5080 (1986); R. Giachetti and V. Tognetti, Phys. Rev. Lett. 55, 912 (1985); (b) L. M. Sesé, Mol. Phys., 78, 1167 (1993); (c) L. M. Sesé, Mol. Phys. 97, 881 (1999).

${ }^{23}$ J. R. Melrose and K. Singer, Mol. Phys. 66, 1203 (1989).

${ }^{24}$ C. Chakravarty, J. Chem. Phys. 116, 8938 (2002); R. Ramírez, C. P. Herrero, A. Antonelli and E. R. Hernández, J. Chem. Phys. 129, 064110 (2008).

${ }^{25}$ L. M. Sesé and R. Ledesma, J. Chem. Phys. 102, 3776 (1995).

${ }^{26}$ L. M. Sesé, J. Chem. Phys. 114, 1732 (2001).

${ }^{27}$ L. M. Sesé, Mol. Phys. 100, 927 (2002).

${ }^{28}$ L. M. Sesé, J. Chem. Phys. 116, 8492 (2002).

${ }^{29}$ L. M. Sesé, Mol. Phys. 101, 1455 (2003).

${ }^{30}$ (a) L. M. Sesé, J. Chem. Phys. 126, 164508 (2007); (b) K. J. Runge and G. V. Chester, Phys.

Rev. B 38, 135 (1988).

${ }^{31}$ L. M. Sesé and L. E. Bailey, J. Chem. Phys. 126, 164509 (2007).

${ }^{32}$ L. M. Sesé, Mol. Phys. 89, 1783 (1996).

${ }^{33}$ L. M. Sesé, Chem. Phys. Lett. 266, 130 (1997).

${ }^{34}$ L. M. Sesé, Mol. Phys. 92, 693 (1997).

${ }^{35}$ L. M. Sesé and R. Ledesma, J. Chem. Phys. 106, 1134 (1997).

${ }^{36}$ L. M. Sesé, J. Chem. Phys. 108, 9086 (1998).

${ }^{37}$ L. M. Sesé and L. E. Bailey, J. Chem. Phys. 119, 10256 (2003).

${ }^{38}$ L. M. Sesé, J. Chem. Phys. 121, 3702 (2004). 
${ }^{39}$ L. E. Bailey and L. M. Sesé, J. Chem. Phys. 121, 10076 (2004).

${ }^{40}$ L. M. Sesé, J. Chem. Phys. 123, 104507 (2005).

${ }^{41}$ L. M. Sesé, J. Phys. Chem. B, 112, 10241 (2008).

${ }^{42}$ L. M. Sesé, J. Chem. Phys. 130, 074504 (2009).

${ }^{43}$ M. P. Allen and D. J. Tildesley, Computer Simulation of Liquids (Oxford, Clarendon, 1989).

${ }^{44}$ J. J. Salacuse, A. R. Denton and P. Egelstaff, Phys. Rev. E 53, 2382 (1996).

${ }^{45}$ W. W. Wood, J. Chem. Phys. 48, 415 (1968); 52, 729 (1970).

${ }^{46}$ (a) J. K. Percus, Phys. Rev. Lett. 8, 462 (1962); L. L. Lee, J. Chem. Phys. 60, 1197 (1974); (c) J. L. Barrat, J. P. Hansen and G. Pastore, Mol. Phys. 63, 747 (1988); (d) R. Evans, in Fundamental of Inhomogeneous Fluids, Chp. 3, Ed. D. Henderson (Marcel Dekker, New York, 1992); (e) A. D. J. Haymet, in Fundamentals of Inhomogeneous Fluids, Chp. 9, Ed. D. Henderson (Marcel Dekker, New York, 1992).

${ }^{47}$ N. Blinov and P.-N. Roy, J. Chem. Phys. 120, 3759 (2004).

${ }^{48}$ K. Shinoda, S. Miura and S. Okazaki, J. Chem. Phys. 114, 7497 (2001).

${ }^{49}$ M. F. Trotter, Proc. Am. Math. Soc. 10, 545 (1959).

${ }^{50}$ G. Jacucci and E. Omerti, J. Chem. Phys. 79, 3051 (1983).

${ }^{51}$ J. Cao and B. J.Berne, J. Chem. Phys. 97, 2382 (1992).

${ }^{52}$ E. de Prunelé, J. Phys. A: Math. Theor. 41, 255305 (2008).

53 (a) X-P. Li and J. Q. Broughton, J. Chem. Phys. 86, 5094 (1987); (b) J. E. Cuervo, P-N. Roy and M. Boninsegni, J. Chem. Phys. 122, 114504 (2005); (c) H. De Raedt and B. De Raedt, Phys. Rev. A 28, 3575 (1983).

${ }^{54}$ R. J. Baxter, Aust. J. Phys. 21, 563 (1968).

${ }^{55}$ M. Dixon and P. Hutchinson, Mol. Phys. 33, 1663 (1977).

${ }^{56}$ T. L. Hill, Statistical Mechanics (Dover, New York, 1987).

${ }^{57}$ Q. Wang, J. K. Johnson and J. Q. Broughton, J. Chem. Phys. 107, 5108 (1997).

${ }^{58}$ A. Baumketner and Y. Hiwatari, Phys. Rev. E. 63, 061201 (2001). 
${ }^{59}$ E. L. Crow, F. A. Davis and M. W. Maxfield, Statistics Manual (Dover, New York, 1960); J. Mandel, The Statistical Analysis of Experimental Data (Dover, New York, 1984).

${ }^{60}$ S.W. Lovesey, Theory of Neutron Scattering from Condensed Matter (Oxford, Clarendon, 1987).

${ }^{61}$ See supplementary material at $[U R L$ will be inserted by AIP] for the complete numerical results of the equations of state.

${ }^{62}$ M. Fierz, Phys. Rev. 106, 412 (1957).

${ }^{63}$ (a) P. C. Hemmer, Physics Lett. A 27, 377 (1968); (b) B. Jancovici, Phys. Rev. 184, 119 (1969);

(c) W. G. Gibson, Mol. Phys. 30, 13 (1975); (d) B. J. Yoon and H. A. Scheraga, J. Chem. Phys. 88, $3923(1988)$.

${ }^{64}$ M. F. Herman, E. J. Bruskin and B. J. Berne, J. Chem. Phys. 76, 5150 (1982).

${ }^{65}$ W. H. Press, B. P. Flannery, S. A. Teukolsky and W. T. Vetterling, Numerical Recipes (Cambridge University Press, Cambridge, 1988).

${ }^{66}$ A. Habenschuss, E. Johnson and A. H. Narten, J. Chem. Phys. 74, 5234 (1981)

${ }^{67}$ P. N. Pusey and W. van Megen, Nature (London) 320, 340 (1986); W. G. Hoover and F. H. Ree, J. Chem. Phys. 49, 3609 (1968). 
TABLE I. Positions, heights and error bars (one-standard deviation) in the vicinitiy of the first peaks ( $v f p$ ) of the instantaneous (ET) and necklace centroid (CM) pair radial correlation functions at selected conditions.

\begin{tabular}{|l|l|l|l|l|l|}
\hline & & \multicolumn{2}{|c|}{ Instantaneous - Eq. (23) } & \multicolumn{2}{c|}{ PI centroid - Eq. (20) } \\
\hline$\lambda_{B}^{*}$ & $\rho_{N}^{*}$ & $r / \AA$ & $g_{E T}(r)-v f p$ & $R / \AA$ & $g_{C M}(R)-v f p$ \\
\hline 0.2 & 0.1 & 4.15 & $1.134 \pm 0.003$ & 4.15 & $1.140 \pm 0.005$ \\
\hline & 0.805 & 3.85 & $3.612 \pm 0.008$ & 3.85 & $4.422 \pm 0.010$ \\
\hline 0.4 & 0.1 & 4.65 & $1.131 \pm 0.002$ & 4.35 & $1.169 \pm 0.005$ \\
\hline & 0.710 & 4.05 & $2.961 \pm 0.005$ & 4.05 & $4.098 \pm 0.012$ \\
\hline 0.6 & 0.1 & 5.10 & $1.129 \pm 0.001$ & 4.75 & $1.187 \pm 0.005$ \\
\hline & 0.595 & 4.35 & $2.437 \pm 0.002$ & 4.25 & $3.430 \pm 0.008$ \\
\hline 0.8 & 0.1 & 5.50 & $1.129 \pm 0.001$ & 5.15 & $1.203 \pm 0.007$ \\
\hline & 0.545 & 4.45 & $2.253 \pm 0.002$ & 4.45 & $3.349 \pm 0.011$ \\
\hline 2 & 0.1 & 6.95 & $1.127 \pm 0.001$ & 6.55 & $1.329 \pm 0.004$ \\
\hline & 0.3 & 5.35 & $1.542 \pm 0.001$ & 5.45 & $2.415 \pm 0.006$ \\
\hline
\end{tabular}


TABLE II. Some typical results of the $B D H+G C$ procedure for the mean $\left\langle h_{C M}(R)\right\rangle \mathrm{CM}$ pair necklace centroid correlations at QHS fluid state point $\left(\lambda_{B}^{*}=0.6, \rho_{N}^{*}=0.5\right)$.

\begin{tabular}{|c|c|c|c|c|c|c|c|c|c|c|c|}
\hline \multicolumn{6}{|c|}{ BDH kernel $(220 \times 220)$} & \multicolumn{6}{|c|}{ BDH kernel $(440 \times 440)$} \\
\hline \multicolumn{2}{|c|}{$m=0$} & \multicolumn{2}{|c|}{$G C(m=1)$} & \multicolumn{2}{|c|}{$G C(m=5)$} & \multicolumn{2}{|c|}{$m=0$} & \multicolumn{2}{|c|}{$G C(m=1)$} & \multicolumn{2}{|c|}{$G C(m=5)$} \\
\hline$R_{Z} / \AA$ & $S_{C M}(k=0)$ & $R_{Z} / \AA$ & $S_{C M}(k=0)$ & $R_{Z} / \AA$ & $S_{C M}(k=0)$ & $R_{Z} / \AA$ & $S_{C M}(k=0)$ & $R_{Z} / \AA$ & $S_{C M}(k=0)$ & $R_{Z} / \AA$ & $S_{C M}(k=0)$ \\
\hline 4.450 & 0.031884 & 4.450 & 0.031887 & 4.450 & 0.031888 & 4.450 & 0.031885 & 4.450 & 0.031887 & 4.450 & 0.031888 \\
\hline 4.857 & 0.032392 & 4.860 & 0.032402 & 4.860 & 0.032402 & 4.857 & 0.032393 & 4.860 & 0.032402 & 4.860 & 0.032402 \\
\hline 5.704 & 0.031546 & 5.695 & 0.031581 & 5.695 & 0.031582 & 5.707 & 0.031517 & 5.697 & 0.031567 & 5.697 & 0.031568 \\
\hline 6.684 & 0.032532 & 6.702 & 0.032655 & 6.702 & 0.032658 & 6.689 & 0.032572 & 6.706 & 0.032712 & 6.707 & 0.032716 \\
\hline 7.604 & 0.031519 & 7.578 & 0.031785 & 7.577 & 0.031795 & 7.609 & 0.031443 & 7.583 & 0.031709 & 7.582 & 0.031717 \\
\hline 8.517 & 0.032128 & 8.573 & 0.032644 & 8.574 & 0.032656 & 8.527 & 0.032252 & 8.582 & 0.032774 & 8.584 & 0.032792 \\
\hline 9.628 & 0.031806 & 9.501 & 0.032431 & 9.498 & 0.032446 & 9.610 & 0.031946 & 9.483 & 0.032579 & 9.479 & 0.032600 \\
\hline 10.239 & 0.031851 & 10.414 & 0.032568 & 10.419 & 0.032585 & 10.261 & 0.032003 & 10.444 & 0.032737 & 10.441 & 0.032761 \\
\hline 11.762 & 0.031444 & 11.613 & 0.032309 & 11.610 & 0.032329 & 11.779 & 0.031218 & 11.787 & 0.032504 & 11.589 & 0.032537 \\
\hline 12.421 & 0.031518 & 12.642 & 0.032492 & 12.648 & 0.032518 & 12.385 & 0.031275 & 12.660 & 0.032724 & 12.669 & 0.032762 \\
\hline & & 13.086 & 0.032491 & 13.079 & 0.032513 & & & 13.054 & 0.032701 & 13.045 & 0.032742 \\
\hline & & 13.301 & 0.032496 & 13.306 & 0.032521 & & & 13.239 & 0.032700 & 13.265 & 0.032744 \\
\hline & & 14.192 & 0.032392 & 14.209 & 0.032427 & & & 13.847 & 0.032659 & 13.749 & 0.032741 \\
\hline & & 14.611 & 0.032403 & 14.426 & 0.032432 & & & 14.423 & 0.032661 & 14.419 & 0.032692 \\
\hline
\end{tabular}


TABLE III. Representative results for the isothermal compressibility of the quantum hard-sphere fluid obtained from the pair centroid correlations for different conditions. $\mathrm{BDH}=$ Baxter-Dixon-Hutchinson method; kernel $(440 \times 440)$. GC $=$ Baumketner- Hiwatari grand canonical corrections. $K=$ number of $R_{Z}$ zeros within the significant range of distances $8.5 \leq R_{Z} / \AA \leq 15.5$. In BDH the cases without zeros in that range are marked with * and the largest $R_{Z}$ is used. Underlined densities mark metastable fluid state points. Error bars are one-standard deviation.

\begin{tabular}{|c|c|c|c|c|c|c|c|}
\hline \multirow[b]{2}{*}{$\lambda_{B}^{*}$} & \multirow[b]{2}{*}{$\rho_{N}^{*}$} & \multicolumn{2}{|c|}{$B D H-\mathrm{GC}(m=0) \quad$ (mean) } & \multicolumn{2}{|c|}{$B D H+G C(m=5) \quad($ mean $)$} & \multicolumn{2}{|c|}{$B D H+G C(m=5) \quad(10$ blocks $)$} \\
\hline & & $K$ & $\left\langle\chi_{T}\right\rangle /\left(10^{-3}{\left.b a r^{-1}\right)}\right.$ & $K^{G C}$ & {$\left[\left\langle\chi_{T}\right\rangle \pm \varepsilon\left(\left\langle\chi_{T}\right\rangle\right)\right] /\left(10^{-3}{b a r^{-1}}^{-1}\right)$} & $\sum_{\alpha} K^{G C}(\alpha)$ & {$\left[\bar{\chi}_{T} \pm s\left(\bar{\chi}_{T}\right)\right] /\left(10^{-3} \mathrm{bar}^{-1}\right)$} \\
\hline \multirow[t]{4}{*}{0.2} & 0.1 & $1 *$ & 84.267 & 11 & $84.512 \pm 0.202$ & 181 & $84.566 \pm 0.037$ \\
\hline & 0.4 & $1 *$ & 4.817 & 17 & $4.747 \pm 0.022$ & 149 & $4.756 \pm 0.005$ \\
\hline & 0.725 & 8 & 0.493 & 4 & $0.525 \pm 0.010$ & 77 & $0.532 \pm 0.001$ \\
\hline & $\underline{0.805}$ & 7 & 0.293 & 6 & $0.310 \pm 0.007$ & 72 & $0.311 \pm 0.001$ \\
\hline \multirow[t]{4}{*}{0.4} & 0.1 & $1^{*}$ & 304.783 & 17 & $304.993 \pm 0.389$ & 159 & $305.168 \pm 0.119$ \\
\hline & 0.4 & $1 *$ & 13.063 & 11 & $13.159 \pm 0.097$ & 131 & $13.209 \pm 0.022$ \\
\hline & 0.625 & 5 & 2.140 & 5 & $2.243 \pm 0.019$ & 92 & $2.244 \pm 0.003$ \\
\hline & $\underline{0.710}$ & 6 & 1.164 & 8 & $1.202 \pm 0.050$ & 70 & $1.222 \pm 0.006$ \\
\hline \multirow[t]{2}{*}{0.6} & 0.1 & $1 *$ & 611.267 & 11 & $615.598 \pm 1.275$ & 184 & $615.435 \pm 0.179$ \\
\hline & 0.3 & $1 *$ & 51.536 & 13 & $52.139 \pm 0.208$ & 161 & $52.093 \pm 0.048$ \\
\hline
\end{tabular}




\begin{tabular}{|c|c|c|c|c|c|c|c|}
\hline \multicolumn{8}{|c|}{ TABLE III. Continued } \\
\hline & 0.525 & 4 & 6.430 & 9 & $6.728 \pm 0.067$ & 76 & $6.695 \pm 0.011$ \\
\hline & $\underline{0.595}$ & 5 & 3.646 & 7 & $3.824 \pm 0.106$ & 75 & $3.868 \pm 0.010$ \\
\hline \multirow[t]{4}{*}{0.8} & 0.1 & 2 & 964.819 & 15 & $975.490 \pm 3.632$ & 167 & $975.807 \pm 0.425$ \\
\hline & 0.3 & 3 & 67.017 & 9 & $68.388 \pm 0.317$ & 140 & $68.348 \pm 0.079$ \\
\hline & 0.5 & 5 & 9.297 & 6 & $9.600 \pm 0.156$ & 94 & $9.559 \pm 0.014$ \\
\hline & $\underline{0.545}$ & 5 & 6.349 & 8 & $6.574 \pm 0.176$ & 72 & $6.623 \pm 0.024$ \\
\hline \multirow[t]{3}{*}{2.0} & 0.1 & 1 & 2575.116 & 6 & $2607.714 \pm 12.630$ & 134 & $2606.590 \pm 1.508$ \\
\hline & 0.2 & 2 & 344.897 & 6 & $354.038 \pm 4.977$ & 105 & $355.738 \pm 0.496$ \\
\hline & 0.3 & 6 & 90.757 & 6 & $91.949 \pm 1.672$ & 70 & $91.718 \pm 0.169$ \\
\hline
\end{tabular}


TABLE IV. Representative results for the QHS fluid equation of state obtained from the PIMC simulations and the centroid pair correlations for different conditions. 1 kpass $=10^{3} N_{S} \times P$ attempted bead moves. $\lambda_{B}^{*} \leq 0.8: N_{S} \times P=500 \times 12$, Kpasses $=800 . \lambda_{B}^{*}=2: N_{S} \times P=432 \times 24$, Kpasses $=1900$. Underlined densities mark metastable fluid state points. Error bars are one-standard deviation.

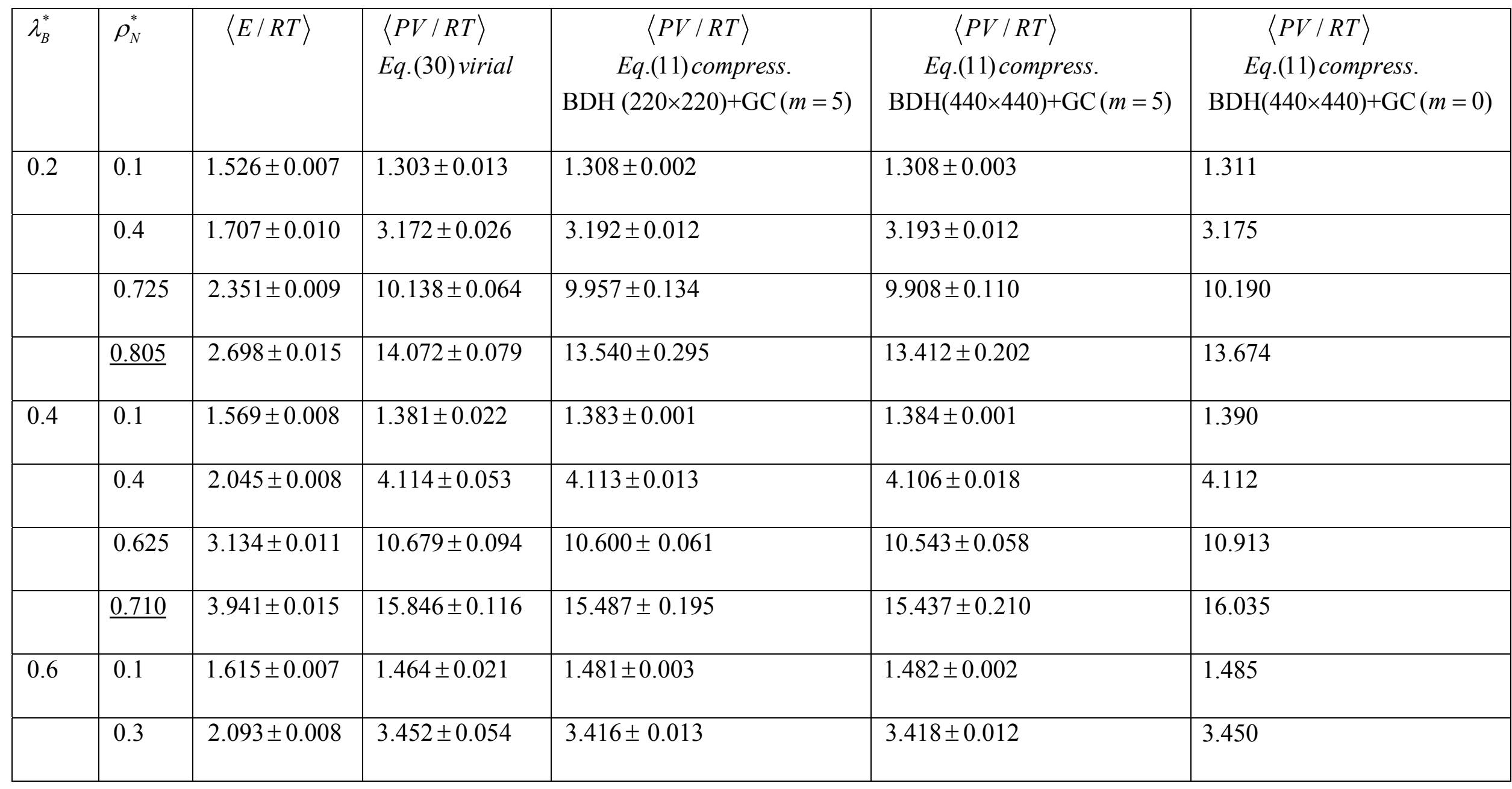




\begin{tabular}{|c|c|c|c|c|c|c|}
\hline \multicolumn{7}{|c|}{ TABLE IV. Continued } \\
\hline \multirow[t]{3}{*}{0.8} & 0.1 & $1.678 \pm 0.006$ & $1.567 \pm 0.031$ & $1.572 \pm 0.003$ & $1.580 \pm 0.002$ & 1.594 \\
\hline & 0.5 & $4.707 \pm 0.014$ & $12.661 \pm 0.166$ & $12.469 \pm 0.230$ & $12.589 \pm 0.078$ & 13.005 \\
\hline & $\underline{0.545}$ & $5.590 \pm 0.016$ & $15.876 \pm 0.191$ & $15.943 \pm 0.316$ & $16.020 \pm 0.189$ & 16.539 \\
\hline 2.0 & 0.1 & $2.382 \pm 0.012$ & $2.624 \pm 0.090$ & $2.742 \pm 0.023$ & $2.789 \pm 0.005$ & 2.792 \\
\hline
\end{tabular}


Figure Captions.

Figure 1.

Necklace centroid direct correlation functions at state point $\left(\lambda_{B}^{*}=2, \rho_{N}^{*}=0.2\right)$ for the first and sixth significant $R_{Z}$ zeros obtained through (BDH+GC).

Figure 2.

Final results $(\mathrm{PIMC}+\mathrm{BDH}+\mathrm{GC})$ for the mean pair radial structures at state point $\left(\lambda_{B}^{*}=2, \rho_{N}^{*}=0.2\right) . \mathrm{ET}=$ instantaneous, $\mathrm{PLR}=$ pair continuous linear response, $\mathrm{CM}=$ necklace centroids. The hard core is located at $\sigma=3.5 \AA$.

Figure 3.

Final results $(\mathrm{BDH}+\mathrm{GC})$ for the Fourier space response functions (static structure factors) at state point $\left(\lambda_{B}^{*}=2, \rho_{N}^{*}=0.2\right) . \mathrm{CM}=$ necklace centroids, $\mathrm{ET}=$ instantaneous, $\mathrm{TLR}=$ total continuous linear response $(\mathrm{PI}=$ path-integral with $\mathrm{P}=24$; $\mathrm{GFH}=$ Gaussian Feynman-Hibbs for self correlations). These functions have been smoothed with B-splines. 

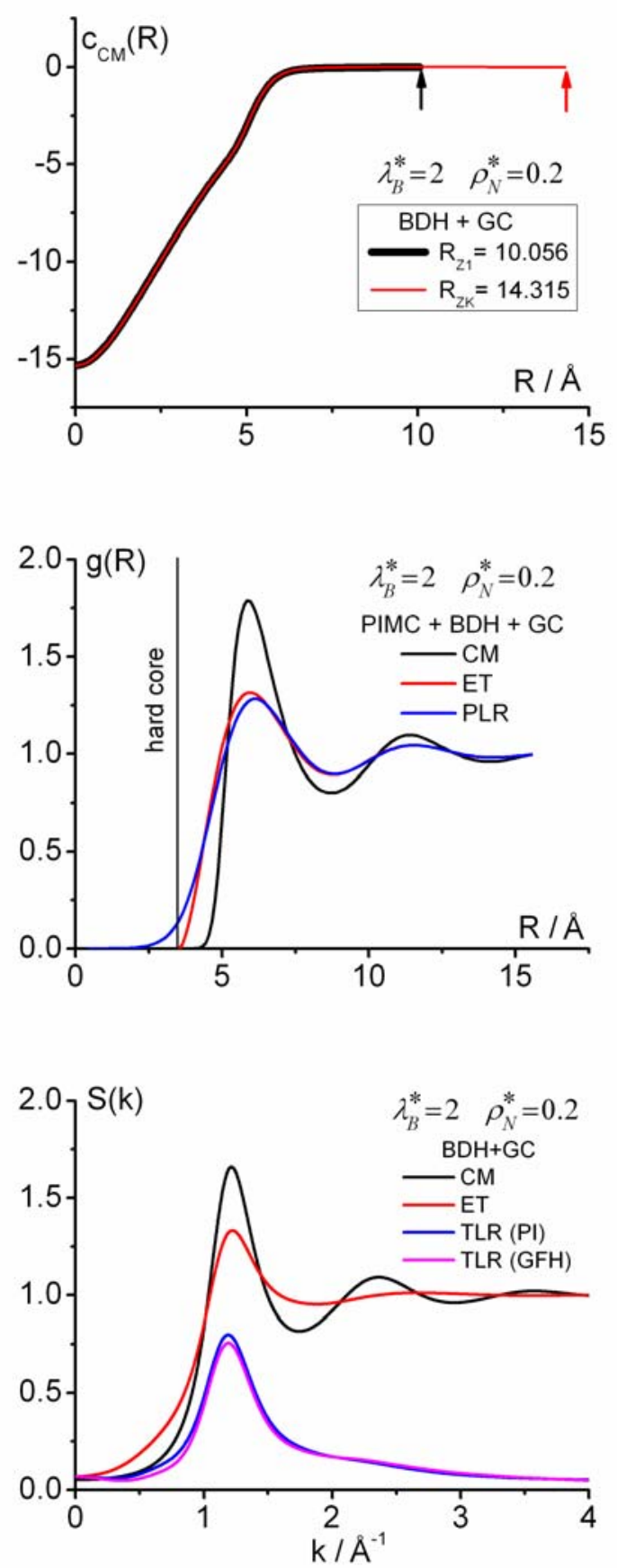